This is the authors' copy of the unpublished version of this manuscript, which is currently under review.

Please cite as:

Masapollo, M., Segawa, J.A., Beal, D., Tourville, J., Nieto-Castañón, A., Heyne, M., Frankford, S., \& Guenther, F.H. (under revision). Behavioral and neural correlates of speech motor sequence learning in neurotypical and stuttering speakers: an fMRI investigation.

\title{
Behavioral and neural correlates of speech motor sequence learning in stuttering and neurotypical speakers: an fMRI investigation
}

Matthew Masapollo $^{1}$, Jennifer A. Segawa ${ }^{1,2}$, Deryk S. Beal ${ }^{1,3}$, Jason A. Tourville $^{1}$, Alfonso Nieto-Castañón ${ }^{1}$, Matthias Heyne ${ }^{1}$, Saul A. Frankford ${ }^{1}$, \& Frank H. Guenther ${ }^{1,4,5}$

${ }^{1}$ Department of Speech, Language and Hearing Sciences, Boston University, Boston, MA 02215

${ }^{2}$ Departments of Neuroscience and Biology, Stonehill College, Easton, MA 02357

${ }^{3}$ Department of Speech-Language Pathology, University of Toronto, Toronto, ON M5G 1V7

${ }^{4}$ Department of Biomedical Engineering, Boston University, Boston, MA 02215

${ }^{5}$ Picower Institute for Learning and Memory, Massachusetts Institute of Technology, Cambridge, MA 02139

To whom correspondence should be addressed. Email: mmasapollo@phhp.ufl.edu

Funding Sources: Research reported in this publication was supported by the National Institute on Deafness and other Communication Disorders of the National Institutes of Health under award number R01DC007683 (F.H. Guenther, PI). The content is solely the responsibility of the authors and does not necessarily represent the official views of the National Institutes of Health. 
NEURAL CORRELATES OF SPEECH MOTOR SEQUENCE LEARNING

\section{Abstract}

2 Stuttering is a neurodevelopmental disorder characterized by impaired production of coordinated

3 articulatory movements needed for fluent speech. It is currently unknown whether these abnormal

4 production characteristics reflect disruptions to brain mechanisms underlying the acquisition

5 and/or execution of speech motor sequences. To dissociate learning and control processes, we used

6 a motor sequence learning paradigm to examine the behavioral and neural correlates of learning

7 to produce novel phoneme sequences in adults who stutter (AWS) and neurotypical controls.

8 Participants intensively practiced producing pseudowords containing non-native consonant

9 clusters (e.g., "gvasf") over two days. The behavioral results indicated that although the two

10 experimental groups showed comparable learning trajectories, AWS performed significantly

11 worse on the task prior to and after speech motor practice. Using functional magnetic resonance

12 imaging (fMRI), the authors compared brain activity during articulation of the practiced words

13 and a set of novel pseudowords (matched in phonetic complexity). FMRI analyses revealed no

14 differences between AWS and controls in cortical or subcortical regions; both groups showed

15 comparable increases in activation in left-lateralized brain areas implicated in phonological

16 working memory and speech motor planning during production of the novel sequences compared

17 to the practiced sequences. Moreover, activation in left-lateralized basal ganglia sites was

18 negatively correlated with in-scanner mean disfluency in AWS. Collectively, these findings

19 demonstrate that AWS exhibit no deficit in constructing new speech motor sequences but do show

20 impaired execution of these sequences before and after they have been acquired and consolidated. 
NEURAL CORRELATES OF SPEECH MOTOR SEQUENCE LEARNING

22 Keywords: speech motor control; motor sequence learning; stuttering; GODIVA model; fMRI 23 
NEURAL CORRELATES OF SPEECH MOTOR SEQUENCE LEARNING

\section{Behavioral and neural correlates of speech motor sequence learning in} stuttering and neurotypical speakers: an fMRI investigation

\section{Introduction}

27 Stuttering is a neurodevelopmental disorder affecting children into adulthood with devastating 28 social effects that impede speech communication. The presenting clinical signs of the disorder 29 include involuntary repetitions and prolongations of phonemes, syllables, or words as well as 30 involuntary silent pauses (Bloodstein \& Ratner, 2008). Despite considerable research, researchers

31 still have a relatively poor understanding of the neural deficits underlying the disorder (see Craig-

32 McQuaide, Akram, Zrinzo, \& Tripoliti, 2014, for a review). Neurobiological accounts of 33 stuttering, such as Alm (2004), Max, Guenther, Gracco, Ghosh, and Wallace (2004), and recently

34 Connally, Ward, Pliatsikas, Finnegan, Jenkinson, Boyles, and Watkins (2018) and Chang and 35 Guenther (2020), propose that stuttering disfluencies arise from an underlying defect in the (left36 lateralized) cortico-basal-ganglia-thalamocortical (cortico-BG) loops (Alexander, DeLong, \&

37 Strick, 1986), which are hypothesized to be responsible for selecting and triggering articulatory 38 motor programs, while suppressing others, at the appropriate time during speech sequencing. There is an extensive body of evidence (recently reviewed by Chang and Guenther [2020]) 40 in favor of the idea that stuttering involves an inability to initiate, sustain, and/or terminate speech

41 motor programs due to anomalous basal ganglia function. For example, numerous acoustic 42 investigations have provided evidence that stuttering involves an impaired ability to transition 43 from the articulatory gestures affiliated with an initial phoneme of a syllable to subsequent 44 gestures, as indicated by protracted voice onset times and formant transition rates (e.g., Yaruss \& 45 Conture, 1993; Bousten, 1995; Robb \& Blomgren, 1997; Robb, Blomgren \& Chen, 1998; Chang, 46 Ohde, \& Conture, 2002). As for the underlying neural circuitry, abnormal functional activity in 47 the basal ganglia has frequently been implicated in stuttering severity (Ingham, Grafton, Bothe, \& 


\section{NEURAL CORRELATES OF SPEECH MOTOR SEQUENCE LEARNING}

Ingham, 2012; Giraud, Neumann, Bachoud-Levi, von Gudenberg, Euler, Lanfermann, \& Preibisch, 2008; Toyomura, Fujii, Kuriki, 2015; Connally et al., 2018). In addition, stuttering-like disfluencies have been reported to emerge in neurodegenerative diseases that impair the function of the basal ganglia, such as Parkinson's disease (Niethammer, Feigin, \& Eidelber, 2012), and deep brain stimulation applied to either the subthalamic nucleus or the globus pallidus pars interna of the basal ganglia can relieve or exacerbate stuttering disfluencies (see Skodda, 2012, and Sapir, 2014, for reviews).

Moreover, and of particular relevance to the current research, some existing theoretical models attribute a role in speech motor sequence learning to the cortico-BG loops (e.g., Kotz \& Schwartze, 2010; Guenther, 2016, chapters 8 \& 10; see also Fee \& Goldberg, 2011, for a similar account in songbird vocal learning). For example, in the Gradient $O$ rder Directions Into Velocities of the Articulators (GODIVA) model of speech sequencing and initiation (Bohland, Bullock, \& Guenther, 2010; Civier, Bullock, Max, \& Guenther, 2013; Guenther, 2016, chapter 8; Segawa, Masapollo, Tong, Smith \& Guenther, 2019), it is proposed that continual speech motor practice leads to the formation of optimized motor programs for frequently occurring kinematic sequences, such as the successive gestures making up a word or syllable. These learned movement "chunks" or "templates" are then encoded and sequentially activated (or "read out") by the cortico-BG loops during production. The fundamental idea is that the cortico-BG loops perform two distinct, but related, cognitive operations during the speech production process; namely, forming regularized motor "chunks" and initiating/activating motor programs affiliated with those "chunks." Results from a functional magnetic resonance imaging (fMRI) study of speech motor sequence learning with neurotypical adults (Segawa, Tourville, Beal, \& Guenther, 2015) provided empirical support for this view. In the initial training phase of the study, participants performed a 


\section{NEURAL CORRELATES OF SPEECH MOTOR SEQUENCE LEARNING}

71 cued motor sequence learning task (outside the scanner) in which they intensively practiced

72 producing pseudowords (monosyllabic $\mathrm{CCVCC}[\mathrm{C}]$, where $\mathrm{C}=$ consonant, $\mathrm{V}=$ vowel) consisting

73 of consonant clusters that were either phonotactically legal in their native language of English

74 (e.g., "blerk") or illegal in English but legal in other natural human languages (e.g., "gvasf”). The

75 results indicated that, behaviorally, motor practice led to measurable performance gains (i.e.,

76 reduced error rates and shorter utterance durations), presumably because the sequences of

77 independent speech movements had become concatenated into larger cohesive "chunks" (see

78 Segawa et al., 2019, for supporting evidence).

79 In the subsequent test phase (inside the scanner), the authors measured the blood-oxygen-

80 level-dependent (BOLD) response during production of the practiced native and practiced non-

81 native words as well as a novel set of non-native words (matched in phonetic complexity) using

82 sparse sampling fMRI (see, e.g., Gracco, Pike, \& Tremblay, 2005). In one set of imaging analyses,

83 BOLD activity associated with production of the novel non-native words was contrasted with that

84 of the practiced non-native words. The results demonstrated increased activation in (left-

85 lateralized) cortical (i.e., pre-supplementary motor area [pre-SMA], anterior insula [aINS], inferior

86 frontal sulcus [IFS], inferior parietal sulcus [IPS], ventral premotor cortex [vPMC]) and

87 subcortical basal ganglia (i.e., globus pallidus [GP]) regions involved in the cortico-BG loops

88 (Alexander et al., 1986). It was further demonstrated that the degree of motor performance

89 improvements between training and test was correlated positively with activity in left aINS. The

90 cortical regions that were found to be recruited in this task overlap with activation foci that have

91 been identified in meta-analyses of working memory neuroimaging studies (see, e.g., Rottschy et

92 al., 2012). Thus, these findings are in keeping with the hypothesis that the cortico-BG loops play

93 an important role in learning and sequencing speech movement "chunks" since a higher number 


\section{NEURAL CORRELATES OF SPEECH MOTOR SEQUENCE LEARNING}

94 of "chunks" will have to be concatenated in a working memory repository (or phonological output

95 store) and then subsequently "read out" during the production of novel sequences compared to

96 practiced ones.

97 In another set of analyses contrasting BOLD activity associated with production of the

98 practiced non-native and practiced native words, the authors found increased activation in a similar

99 network of brain areas, although notably no increased activation was found in left GP for this

100 contrast. They did, however, report increased activation in right cerebellum (lobule VI) for this

101 contrast, suggesting that other subcortical structures that extend beyond the cortico-BG may also

102 play a role in speech motor sequencing learning and motor control (a point we will return to in the

103 general discussion).

104 Nevertheless, if the cortico-BG loops are involved in successively encoding and activating

105 speech motor "chunks" during sequence learning and production, then that raises the possibility

106 that the neural deficits hypothesized to underlie stuttering are related not only to problems with

107 motor execution, but also to impaired motor learning mechanisms. Consistent with this view are

108 experimental findings, in the behavioral literature, that adults who stutter (AWS) and adults with

109 normal speech (ANS) often show differences in their ability to learn a variety of novel speech and

110 non-speech (i.e., finger-tapping) movement sequences (e.g., Ludlow, Siren, \& Zikria, 1997; Smits-

111 Bandstra, De Nil, \& Saint-Cyr, 2006; Namasivayam \& van Lieshout, 2008). Qualitatively similar

112 findings have also been found in patients with Parkinson's disease (Ferraro, Balota, \& Connor,

113 1993; Jackson et al., 1995; Helmuth, Mayr, \& Daum, 2000; Vakil et al., 2000; Smits-Bandstra \&

114 Gracco, 2013; Mollaei, Shiller, \& Gracco, 2013).

115 In the current research, we performed a functional brain-imaging study to distinguish

116 between motor execution and motor learning impairments in AWS at both the behavioral and 


\section{NEURAL CORRELATES OF SPEECH MOTOR SEQUENCE LEARNING}

117 neural levels, by replicating and extending Segawa et al. (2015) with a cohort of adult speakers

118 with persistent developmental stuttering. Towards this end, we trained AWS and ANS to produce

119 pseudowords containing non-native consonant clusters. Participants were first trained over several

120 days (outside the scanner) to produce two sets of novel CCVCC pseudowords: (1) syllables that

121 involved native consonant clusters (practiced native) and (2) syllables comprised of non-native

122 consonant clusters that are phonotactically illegal in English (practiced non-native). Based on

123 previous results (Segawa et al., 2015; see also Segawa et al., 2019), we expected to observe

124 significantly larger performance gains throughout the course of training for the practiced non-

125 native words than for the practiced native words (for which performance is already expected to be

126 near ceiling at the beginning of training). During a subsequent testing phase (inside the scanner),

127 we then contrasted BOLD activity associated with production of the practiced non-native and

128 practiced native words, and the practiced non-native and practiced native sequences.

129 We hypothesized that, if motor sequence learning mechanisms per se are impaired in 130 stuttering, then: 1) At the behavioral level, AWS should show a slower rate of learning over the 131 course of training and/or poorer learning outcomes compared to ANS; and 2) At the neural level, 132 contrasting the BOLD signal for the novel non-native - practiced non-native and/or practiced non133 native - practiced native conditions should yield less activity in the regions of the cortico-BG 134 circuit previously identified in Segawa et al. (2015) in AWS, especially left aINS, since they will 135 not have fully formed cohesive motor "chunks" for the trained speech sequences.

136 If, however, the only core deficit underlying stuttering is related to motor implementation 137 rather than to motor learning mechanisms, then: 1) At the behavioral level, AWS should show 138 comparable performance gains over time but will display poorer (and/or slower) overall motor 139 performance compared to ANS; and 2) At the neural level, AWS should show a similar reduction 


\section{NEURAL CORRELATES OF SPEECH MOTOR SEQUENCE LEARNING}

140 in activations in the aforementioned regions of the cortico-BG circuit implicated in motor

141 sequences learning and working memory (left aINS, pre-SMA, and IFS) as ANS, but should still

142 show differences in primary motor (and possibly premotor) cortex; and 3) the size of the BOLD

143 activity difference in (at least some of) these brain areas will covary with stuttering severity (and/or

144 in-scanner disfluency) since mechanisms involved in selecting and initiating successive speech

145 motor programs will be more impaired in AWS with more severe stuttering.

\section{2. Material and Methods}

\subsection{Participants}

148 Sixteen adults who stutter (AWS; 14 male, age range: 18-42, median age: 26) and fifteen adults

149 who do not stutter (ANS; 13 male, age range: 18-40, median age: 25 ) were paid for participating

150 in three testing sessions on three separate days. Participants reported normal (or-corrected-to-

151 normal) vision and no history of hearing, speech, language or neurological deficits (apart from

152 stuttering in the AWS group). All were right-handed (Oldfield, 1971; mean score $=80.46$ ) and

153 native speakers of American English with no previous experience with any of the languages used

154 in stimulus creation (see following text). Individuals were excluded from taking part in the study

155 if they were currently on medications that may have substantial effects on neural activity, or had

156 claustrophobia preventing them from completing the MRI protocol. All participants underwent a

157 magnetic resonance safety screening.

158 An experimenter interviewed all participants to confirm the diagnosis of persistent

159 developmental stuttering in AWS and to confirm normal speech production in ANS. None of the

160 AWS were enrolled in a fluency-shaping program at the time of participation. The stuttering

161 severity of each AWS was assessed using the Stuttering Severity Instrument, Edition 4 (SSI-4;

162 Riley, 2008). As part of this assessment, each AWS was video recorded while reading aloud, 


\section{NEURAL CORRELATES OF SPEECH MOTOR SEQUENCE LEARNING}

163 conversing with an experimenter, and speaking on the telephone. A certified speech-language

164 pathologist then rated the frequency and durations of the stuttering events and the presence of

165 physical concomitants that accompanied the moments of disfluency (e.g., eye-blinking). Stuttering

166 severity in the AWS group ranged from 13 to 48, with a median of 27 and an interquartile range

167 of 17-34. Five of the sixteen AWS were categorized as "very mild" (SSI-4 score $<17$ ), 1 as "mild"

168 (18-24), 5 as "moderate" (25-31), 3 as "severe" (32-36), and 2 as "very severe" (37-46).

$169 \quad 2.2$ Stimuli

170 The speech stimuli consisted of several sets of monosyllabic pseudoword sequences (15

171 words/set). As shown in Table 1, all items contained either native or non-native syllable-initial

172 (onset) and syllable-final (coda) consonant clusters. In the native sequences (e.g., BLERK,

173 THRIMF, TRALF), the onset and coda clusters are phonotactically legal in English; in the non-

174 native sequences (e.g., FPESHCH, GVASF, TPIPF), the clusters are phonotactically illegal in

175 English, but do occur in some other natural human language. The non-native clusters were taken

176 from a variety of languages and language families including Hebrew, Leti, Taba, Romani, Polish,

177 Lithuanian, Romanian, Georgian, Tepehua, Hungarian, and Pima; participants reported no prior

178 experience with any languages in which these consonant clusters readily occur. None of the stimuli

179 were an orthographic or a phonological word according to the MRC Psycholinguistic Database

180 (Coltheart, 1981). All items containing non-native clusters had a neighborhood size of 0 , and no

181 real English words could be created by adding, deleting, or substituting a single phoneme in any

182 subsequence. All of the clusters - both native and non-native alike - were bi- or tri-consonantal

183 and used in either onset position or coda position, but not both. Each cluster was used in only one

184 word (i.e., no two words contained the same consonant cluster). The number of phonemes per

185 word was counter-balanced across experimental conditions (see below). 
NEURAL CORRELATES OF SPEECH MOTOR SEQUENCE LEARNING

\section{-- [Insert Table 1 about here] --}

To create the prompts for the elicited production task, a female native speaker of American English was recorded producing the words. The model speaker was phonetically trained and had previously practiced producing the sequences until each stimulus could be executed fluently (i.e., without vocoid epenthesis or phoneme omissions, swaps, or substitutions). All recordings took place in a sound-attenuated booth. The speech was recorded directly to a computer using Audacity ${ }^{\circledR}$ software (Version 2.0.3, Audacity Team) via a microphone (Samson C01U studio condenser) connected to a pre-amplifier (44.1-kHz sampling rate, 32-bit quantization). The speaker recorded multiple randomized repetitions of each token. From these repetitions, one instance of each token was selected on the basis of clarity and acoustic similarity in voice pitch $(f 0)$ to the other stimuli in the set. Using Praat software (Boersma \& Weenink, 2020), all recorded tokens were digitally edited to be matched for peak intensity and duration (i.e., $480 \mathrm{~ms}$ ) without changing $f 0$.

\subsection{Procedure and Design}

Participants completed a cued sequence production task (see, e.g., Segawa et al., 2015, 2019). The experiment consisted of a training phase, during which participants learned to produce 15 words containing native clusters and 15 words containing non-native clusters, followed by a test phase, during which participants were tested on their ability to produce all 30 of the previously learned words and 15 novel words containing non-native consonant clusters. FMRI data were only collected during the test phase (see below). For both phases, participants were asked to repeat aloud each of the target words individually, which were presented both auditorily over noisecancelling headphones and visually using text stimuli (as shown in Table 1). The training phase consisted of eight blocks of trials over two consecutive days (four on day one and four on day 


\section{NEURAL CORRELATES OF SPEECH MOTOR SEQUENCE LEARNING}

209 two). Each training block contained eight repetitions of each word, for a total of 240 trials per

210 block. The test phase consisted of eight blocks of trials performed on day three (inside the MRI

211 scanner) after completing the training phase (outside the scanner on days one and two). Each test

212 block contained five to six repetitions of each word, for an average of 40 trials per block, from

213 three stimulus categories (conditions): (1) practiced native words (i.e., words comprising native

214 clusters that were previously encountered in the training phase), (2) practiced non-native words

215 (i.e., words comprising non-native clusters that were previously encountered in the training phase),

216 and (3) novel non-native words (i.e., novel words with novel clusters that were not encountered in

217 the training phase).

218 Participants were divided into four groups, each of which practiced producing a different

219 subset of the native and non-native sequences during training. The non-native words that were not

220 practiced during training were used as novel non-native words during the imaging session.

221 Assignment of non-native words to the practiced non-native and novel non-native categories was

222 counterbalanced across participants. The training phase occurred one to two days before the test

223 phase to allow for sleep-mediated memory consolidation of the newly acquired motor traces (see,

224 e.g., Fenn, Nusbaum, \& Margoliash, 2003; Doyon, Bellec, Amsel, Penhune, Monchi, Carrier,

225 Lehéricy \& Benali, 2009; Doyon, Albouy, Vahdat, \& King, 2015; Vahdat, Fogel, Benali, \&

226 Doyon, 2017; cf. Brawn, Fenn, Nusbaum, \& Margoliash, 2010; Pan \& Rickard, 2015).

227 During training, participants were seated in a chair in front of a laptop (IBM Lenovo

228 ThinkPad X61s) computer screen in a sound-treated laboratory room that was dimly lit. The 229 auditory speech stimuli were presented over headphones (Sennheiser, HD 280 Pro) at a

230 comfortable listening level and utterances produced by the participants were recorded with a

231 Samson (Hauppauge, NY) C01U USB studio condenser microphone connected to the computer 


\section{NEURAL CORRELATES OF SPEECH MOTOR SEQUENCE LEARNING}

232 via a MOTU microbook audio interface. Utterances were recorded using MATLAB (MathWorks

233 Inc., Natick, MA) at $44.1 \mathrm{kHz}$. During test, participants laid supine in an MRI scanner. Instructions

234 and visual stimuli were projected onto a screen viewed from within the scanner via a mirror

235 attached to the head coil. The auditory stimuli were played over Sensimetrics model S-14 MRI-

236 compatible earphones. Participants' productions were transduced by a Fibersound model FOM1-

237 MR-30m fiber-optic microphone, sent to a Lenovo ThinkPad X61s, and recorded using MATLAB 238 at $44.1 \mathrm{kHz}$.

239 The trial structure was identical during training and test. First, the orthographic display of

240 a given syllable was centrally presented in tandem with its corresponding auditory prompt.

241 Participants only heard each prompt once on each trial. Then, after the offset of the auditory

242 presentation, a tone was presented for $50 \mathrm{~ms}$. The time between stimulus offset and tone onset was

243 randomly jittered between 500 and 1,000 ms. This tone served as a "GO" signal that prompted the

244 participant to go ahead and repeat the token as clearly and accurately as possible. For all phases,

245 participants produced the target syllables in a pseudorandom order. The combination of the

246 auditory and orthographic presentations was necessary because prior studies have shown that

247 listeners tend to perceive non-native consonant clusters as epenthesized disyllabic sequences (e.g.,

248 Pitt, 1998; Dupoux, Kakehi, Pallier, Hirose, \& Mehler, 1999; Berent, Steriade, Lennertz, \&

249 Vaknin, 2007; Dupoux, Parlato, Frota, Hirose, \& Peperkamp, 2011; Davidson \& Shaw, 2012).

250 Moreover, in another study that directly examined the effects of stimulus input modality (audio

251 only vs. audio and text) on speakers' ability to produce non-native consonant clusters (Davidson,

252 2010), it was found that the presence of text led to an improvement in overall task performance.

253 For both phases, participants were instructed to repeat the target syllable as clearly and

254 accurately as possible as soon as they heard the tone. Several familiarization trials with 


\section{NEURAL CORRELATES OF SPEECH MOTOR SEQUENCE LEARNING}

255 experimenter feedback were included at the start of the experiment to confirm that participants

256 understood the task instructions and were able to perform the task. The sequences used during

257 these initial practice trials were not used at any point in the rest of the study. Feedback was not

258 provided during the actual training or test phases.

2592.4 Behavioral Data Analysis

260 We conducted several analyses to provide evidence, at the behavioral level, of incremental motor

261 learning throughout the two days of speech motor practice (outside the MRI scanner), and that

262 such learning was retained at test (inside the scanner). In the first analysis, we analyzed three

263 temporally sensitive measures of learning across the eight training blocks (on days 1 and 2): (1)

264 articulatory sequencing error rate, (2) utterance duration (interval from utterance onset to offset),

265 and (3) reaction time (RT) (interval from the offset of the go-signal to utterance onset). These

266 measures are generally believed to quantify the ease or difficulty with which speakers retrieve and

267 execute speech sequences (Sternberg, Monsell, Knoll, \& Wright, 1978) and are commonly used in

268 both the motor sequence learning and second language learning literatures as measures of learning

269 extent (see, e.g., Rauschecker et al., 2008; Nakamura, Sakai, \& Hikosaka, 1998; Brawn et al.,

270 2010; Doyon et al., 2015; Buchwald et al., 2019; Segawa et al., 2019). Sequencing errors were

271 defined as phoneme additions (including schwa insertions), deletions, and substitutions, and

272 utterance repetitions, omissions, and restarts. Based on prior findings reported with neurotypical

273 speakers (Segawa et al., 2015, 2019), we expected to observe greater learning (at least in the ANS

274 group) for the non-native sequences because those sequences included both novel words and novel

275 onset and coda clusters, whereas the native sequences included novel words with familiar onset

276 and coda clusters. 


\section{NEURAL CORRELATES OF SPEECH MOTOR SEQUENCE LEARNING}

In the second analysis, to provide evidence of the retention of learning at test, we examined 278 the same three performance measures for each experimental group (ANS vs. AWS) and condition

279 (practiced native vs. practiced non-native vs. novel non-native) during the test phase (on day 3 ).

280 For each word production, each measure was calculated following the removal of noise associated

281 with the scanner bore echo and peripheral equipment using a Wiener filter (Wiener, 1949). The

282 coder was blind to the condition (practiced vs. novel) of the non-native syllables. We hypothesized

283 that if participants formed regularized motor "chunks" for the previously trained speech sequences,

284 then they should perform more accurately (and/or faster) at generating the practiced native and 285 non-native sequences compared to the novel non-native sequences.

286 In a final analysis, we examined whether, and if so, how, speech motor practice influenced

287 fluency in the AWS group. Toward this end, we compared the mean number of trials perceptually 288 coded as containing at least one stuttering-like disfluency during both the training and test phases 289 (for the AWS group only) for each stimulus condition (practiced native vs. practiced non-native 290 vs. novel non-native). Disfluencies were determined by a certified speech-language pathologist.

291 For each of these analyses, custom MATLAB software (Natick, MA) was used to 292 perceptually rate and acoustically measure onsets and offsets of syllables by viewing the waveform 293 and spectrogram and listening to the audio files. Listeners first marked, blinded to experimental 294 condition, whether the target phoneme sequence was produced fluently. Disfluent productions 295 were further categorized into four possible disfluency types: (1) repetition (i.e., a phoneme or 296 syllable was repeated); (2) prolongation (i.e., a phoneme was prolonged); (3) audible or silent 297 blocking (involuntary filled or unfilled pauses in speaking), and (4) clustered (i.e., more than one 298 stutter type was produced for a given sequence). 


\section{NEURAL CORRELATES OF SPEECH MOTOR SEQUENCE LEARNING}

Each utterance was then marked for four possible sequencing error subtypes: (1) approximation of the target (i.e., phoneme deletion/omission, insertion, substitution, vowel epenthesis); (2) unrecognizable from target (i.e., an entirely different sequence was produced); (3)

302 unfinished word (i.e., the sequence was produced by was not complete before the end of the 303 recording window); and (4) silence (i.e., no sequence was produced at the time of recording). Since

304 we were concerned with the learning of non-native phonotactics rather than sub-phonemic 305 allophonic details, productions from either the model speaker or the participants were not judged on how natural they sounded in the language from which they were derived. "silent" pauses or blocks associated with stuttering from intentional speech onset delays with the audio-only recordings that we obtained in the scanner (although there were constraints on what the

311 trials with stuttering pauses were categorized as sequencing error subtype 4 (i.e., no sequence

312 produced). ${ }^{1}$ This is a limitation inherent in the current study and all studies of this kind in stuttering

313 (unless video recordings of articulatory behavior inside the scanner are obtained).

314 Mean disfluency rates for each subject were calculated as the percentage of trials that

315 contained one or more disfluency error sub-types, and mean error rates for each subject were

316 calculated as the percentage of trials that contained one or more error subtypes. For each

317 production containing no sequencing or disfluency errors, utterance onset and offset were

318 automatically labeled based on sound pressure level thresholds, then hand-checked. Note that, in

\footnotetext{
${ }^{1}$ In order to explore the possibility that some trials containing "silent" stuttering blocks were included in the neuroimaging analyses, we plotted histograms showing the distribution of RT scores for each stuttering speaker in the test phase (inside the scanner). Critically, these plots did not reveal a bimodal distribution with one peak having frequency values clustered to the right. This finding suggests that any potential blocks or pauses were likely categorized as silent errors (sequencing subtype 4 as described above) and were excluded from the imaging analyses, in which case, the BOLD responses would not have been contaminated by potential delays in RT. See Supplementary Materials for further details.
} 


\section{NEURAL CORRELATES OF SPEECH MOTOR SEQUENCE LEARNING}

319 the neuroimaging component of the study (described below), only trials in which participants

320 produced the target sequence accurately and fluently were analyzed.

3212.5 fMRI Paradigm

322 In addition to the three speaking conditions (practiced native vs. practiced non-native vs. novel

323 non-native), a silent baseline condition was intermixed during imaging in which participants

324 viewed a series of asterisks on the screen instead of the orthographic stimulus and rested quietly

325 instead of uttering a word. FMRI data were acquired using a sparse sampling protocol (see, Belin,

326 Zatorre, Hoge, Evans, \& Pike, 1999; Gracco et al., 2005; Perrachione \& Ghosh, 2013) that allowed

327 participants to produce the target syllables during silent intervals between volume acquisitions. A

328 single volume was acquired approximately $4 \mathrm{sec}$ after speech onset on each trial which aligns with

329 the 4-6 second delay in peak BOLD response onset (Belin et al., 1999). By scanning between

330 speech productions, this protocol avoids the influence of scanner noise on speaker performance

331 and brain activity responses and image artifacts resulting from speech-induced motion of the head.

332 The cued sequence paradigm was identical to that used during training (outside of the

333 scanner) except with an additional pause after the production of each syllable to temporally align

334 the image acquisition to the expected peak of the hemodynamic response. As previously described,

335 the test phase consisted of eight blocks of trials which corresponded to eight functional runs. A

336 single volume was recorded on each trial and the delay between volumes was approximately 10

337 seconds. Each functional run contained five or six productions of each stimulus item, for a total of

33840 trials per run (each run lasted approximately 6-7 minutes). This resulted in a total of 320 test

339 trials (80 trials per condition, including baseline). Conditions were shuffled and pseudorandomly

340 distributed across the eight functional runs with at least eight instances of each condition appearing

341 in each run. 


\section{NEURAL CORRELATES OF SPEECH MOTOR SEQUENCE LEARNING}

\subsection{Image Acquisition}

All neuro-imaging data were acquired using a 3-Tesla Siemens TIM Trio scanner, equipped with a 32-channel head coil. T2*-weighted gradient echo echo-planar fMRI images were collected to assess blood-oxygen-level-dependent (BOLD) responses during the test phase. 41 horizontal slices were collected in each functional volume (in-plane resolution $=3.1 \mathrm{~mm}^{2}$, slice thickness $=3 \mathrm{~mm}$, gap $=25 \%$, acquisition time $=2.5 \mathrm{sec}, \mathrm{TE}=20 \mathrm{msec}$ ); volumes were automatically aligned to the AC-PC line. Prior to collecting functional data, a gradient-echo field map sequence was collected; the resulting magnitude and phase images was enabled offline correction of magnetic field distortions in functional images during data preprocessing (see below; Jezzard \& Balaban, 1995). Structural images were collected using a T1-weighted multi-echo MPRAGE pulse sequence $\left(\right.$ MEMPRAGE, voxel size $=1 \mathrm{~mm}^{3}, 176$ axial slices, $256 \times 256 \mathrm{FOV}$, repetition time $[\mathrm{TR}]=2530$ msec, echo time $[\mathrm{TE}]=3.44 \mathrm{~ms}$, flip angle $=7^{\circ}$ ).

\section{6 fMRI Data Analysis}

Image preprocessing. Functional data were processed using tools from the following software packages that were integrated into a processing stream within SPM12 (Statistical Parametric Mapping, v12; www.fil.ion.ucl.ac.uk/spm/): FreeSurfer (Fischl et al., 2002; Dale, Fischl, \& Sereno, 1999; Fischl, Sereno, \& Dale, 1999; www.freesurfer.net), Artifact Detection Tools (ART; www.nitrc.org/projects/artifact_detect/), and the CONN toolbox (Whitfield-Gabrieli \& Nieto-Castañón, 2012). Freesurfer was used to remove non-brain components of the T1 structural volumes, segment the brain into gray matter, white matter, and cerebral spinal fluid components, generate a reconstruction of the cortical surfaces of each hemisphere and to identify cortical and subcortical regions of interest (ROIs) (see below). Functional data were preprocessed through two pipelines: a surface/vertex-based pipeline for analysis of cortical responses and a 


\section{NEURAL CORRELATES OF SPEECH MOTOR SEQUENCE LEARNING}

365 volume/voxel-based pipeline for analysis of subcortical basal ganglia and cerebellar

366 responses. Prior to pre-processing, the first volume of each functional series was removed because

367 it served only as a trigger for the initial experimental trial.

368 A surface/vertex-based analysis pipeline was used to assess BOLD response differences

369 for each experimental group and for each contrast in the cerebral cortex. Functional images from

370 each subject were simultaneously realigned to the mean subject image and unwarped (motion-by-

371 inhomogeneity interactions) using the SPM12 realign and unwarp procedure (Andersson, Hutton,

372 Ashburner, Turner, \& Friston, 2001). Outlier scans were detected using ART based on framewise

373 displacement (scan-to-scan motion threshold of $0.9 \mathrm{~mm}$ ) and mean signal change (scan-to-scan

374 signal change threshold of 5 standard deviations above the mean (see Nieto-Castañón, 2020 for

375 details $^{2}$ ). Functional volumes from each subject were then co-registered with their high-resolution

376 T1 structural images and resliced using SPM12's inter-modal registration procedure with a

377 normalized mutual information objective function. The functional data were then resampled at the

378 location of the FreeSurfer fsaverage level-8 tessellation (163,842 vertices and 327,680 faces)

379 projected on each subject-specific cortical surface, averaged across 10 intervals along the normal

380 between the white matter and pial surfaces, and smoothed using iterative diffusion smoothing with

381 a series of 40 discrete steps, approximately equivalent to a $8 \mathrm{~mm}$ full-width half-maximum

382 (FWHM) two-dimensional Gaussian smoothing kernel (Hagler et al. 2006, Nieto-Castañón, 2020).

383 A volume/voxel-based analysis pipeline was used to identify differences in subcortical

384 BOLD responses. Following the realignment, unwarping steps described above, functional

385 volumes, and the original T1 structural volumes, were simultaneously segmented and normalized

\footnotetext{
${ }^{2}$ Framewise displacement is computed at each timepoint by considering a $140 \times 180 \times 115 \mathrm{~mm}$ bounding box around the brain and estimating the largest displacement among six control points placed at the center of this bounding-box faces. Global BOLD signal change is computed at each timepoint as the change in average BOLD signal within SPM's global-mean mask scaled to standard deviation units.
} 


\section{NEURAL CORRELATES OF SPEECH MOTOR SEQUENCE LEARNING}

386 directly to Montreal Neurological Institute (MNI) space using SPM12's combined normalization

387 and segmentation procedure (Ashburner \& Friston, 2005). Prior to MNI-normalization, both the

388 functional and anatomical volume origins were centered to coordinates $[0,0,0]$ in order to improve

389 the quality of the iterative procedure initial starting estimates. Functional volumes were then

390 spatially smoothed using an $8 \mathrm{~mm}$ FWHM Gaussian kernel in order to increase BOLD signal-to-

391 noise ratio and reduce the influence of residual variability in functional and gyral anatomy across

392 subjects (Nieto-Castañón, 2020).

Subject-level BOLD contrast analyses. After preprocessing, BOLD responses were

394 estimated using a general linear model (GLM) in SPM 12. Because functional volumes were

395 acquired in a sparse sequence, the hemodynamic response function for each stimulus event was

396 modeled as a finite impulse response. The model included four condition-specific variables

397 (practiced native, practiced non-native, novel non-native, and baseline). Trials with productions

398 that were perceptually rated as containing either a sequencing error (e.g., phoneme deletions,

399 insertions or substitutions) or a stuttering-like disfluency (e.g., phoneme/syllable repetitions,

400 prolongations, or blocks), or were found to be outliers by ART were modeled as separate

401 conditions (one individual regressor per trial) thereby removing variability resulting from these

402 trials from the effects- and contrast-of-interest estimates. For each individual run, regressors were

403 added to the model to remove linear effects of time (e.g. signal drift, adaptation) and the six motion

404 covariates (taken from the realignment step) and a constant term.

405 The model was estimated at each vertex (surface-based analysis) or voxel (volume-based)

406 analysis for each participant, resulting in two surface maps (one for each hemisphere) and a volume

407 map of the model regressor coefficients for each condition. These condition estimates were then

408 contrasted to yield effect-size maps of the following contrasts of interest: differences in the 


\section{NEURAL CORRELATES OF SPEECH MOTOR SEQUENCE LEARNING}

409 response during novel non-native and practiced non-native conditions (novel non-native -

410 practiced non-native), and differences in the response during the practiced non-native and

411 practiced native conditions (practiced non-native - practiced native).

412 Group-level analyses. Group-level $t$-statistics were calculated separately for each contrast

413 map. Cluster-level inferences were based on Threshold Free Cluster Enhancement (TFCE; Smith

414 and Nichols, 2009) with default parameters $\mathrm{E}=0.5$ and $\mathrm{H}=2$. This method assigns TFCE scores to

415 each voxel characterizing the amount of cluster-like local spatial support at each location. These

416 scores are then compared to their expected distribution under the null-hypothesis, estimated using

4171000 randomization/permutation iterations and results are thresholded at Family-wise Error false

418 positive probability $\left(\mathrm{p}_{\mathrm{FWE}}\right)<0.025$. This threshold was used as a conservative means to ensure a

419 cluster-level $\mathrm{p}_{\mathrm{FWE}}<0.05$ across the cortical surface and subcortical volume analyses.

$420 \quad 2.7$ Region-of-interest-based analyses

421 To increase statistical power and sensitivity, we supplemented the surface/vertex-based and 422 volume/voxel-based analyses with region-of-interest (ROI) analyses (Nieto-Castañón, Ghosh, 423 Tourville, \& Guenther, 2003) based on a priori hypotheses derived from the results of Segawa et 424 al. (2015). Specifically, we used anatomically-defined ROIs in the speech production network that 425 overlapped areas of significant BOLD activity in Segawa et al. (2015) for each contrast of interest. 426 The predefined cortical and subcortical (basal ganglia and cerebellar) ROIs are listed in Table 2; a 427 detailed description of the anatomical landmarks used for cortical parcellation are provided in 428 Tourville and Guenther (2003). For the novel non-native-practiced non-native contrast, we tested 429 whether brain activity was significantly different in globus pallidus internal (GPi) and external 430 (GPe) segment ROIs; these ROIs were derived from the probabilistic atlas of the basal ganglia 431 described by Keuken and colleagues (2014). For the practiced non-native - practiced native 


\section{NEURAL CORRELATES OF SPEECH MOTOR SEQUENCE LEARNING}

432 contrast, we tested whether brain activity was significantly different in right cerebellum lobule VI,

433 which was derived from the SUIT probabilistic atlas of the cerebellum (Diedrichsen et al., 2009).

434 Affirmation of differences in these ROIs would replicate the Segawa et al. (2015) findings with

435 neurotypical speakers.

$436 \quad 2.8$ Brain-behavior correlation analyses

437 Two types of hypothesis-driven analyses were conducted to identify potential relationships 438 between behavioral measures and brain activity. In the first type of analysis, we conducted multiple 439 correlation tests to identify relationships between behavioral measures of motor sequence learning 440 success and BOLD activity. Specifically, we tested for correlations between (i) the mean BOLD 441 response in the cortical and subcortical ROIs that showed task-activated clusters in Segawa et al. 442 (2015; Table 2) in both the novel non-native - practice non-native and the practiced non-native 443 practiced native contrasts and (ii) each of the three motor learning indices (error rate, utterance 444 duration, and RT). No correction was applied for the number of ROIs in this ROI list. FreeSurfer 445 was used to define the ROIs on each individual cortical surface using the labeling system described 446 in Cai, Tourville, Beal, Perkell, Guenther, and Ghosh (2014). These ROIs included left-lateralized 447 aINS, vPMC, anterior frontal operculum [aFO], preSMA, IFS, posterior superior temporal sulcus $448[\mathrm{pSTs}]$, and planum temporale [PT], as well as left GPi and GPe. For each participant, we 449 computed each motor learning index by subtracting the mean performance difference between the 450 novel non-native and practiced non-native productions. We normalized for differences between 451 participants by dividing these differences by the practiced non-native syllable measure, on a per452 participant basis. For example, the utterance duration learning measure was the mean duration 453 difference between the novel non-native and practiced non-native productions divided by mean 


\section{NEURAL CORRELATES OF SPEECH MOTOR SEQUENCE LEARNING}

454 duration of the practiced non-native productions. Each motor learning index was then correlated

455 with the mean beta coefficient within each significant cluster from each contrast.

456 In the second type of analysis, SSI measures (Riley, 2008) of stuttering frequency and

457 overall severity (described above) were used, in addition to the number of disfluencies produced

458 during the test phase (inside the scanner), to identify potential relations between stuttering-related

459 behaviors and brain activity. Specifically, we tested for correlations in the AWS group only with

460 the mean composite SSI scores and mean disfluency rates during test (inside the scanner) and the

461 mean BOLD responses in 8 hypothesis-based ROIs within the cortico-BG loops (listed in Table

462 2) in the novel non-native - practice non-native contrast (based on fluent trials only). These ROIs

463 included left premotor and primary motor areas (ventral and mid premotor cortices [vPMC,

464 midPMC] + ventral and mid motor cortices [vMC, midMC]), left medial prefrontal areas (left

465 supplementary motor area [SMA] + preSMA), as well as several left-lateralized subcortical basal

466 ganglia sites (GPi, GPe, caudate, putamen, ventral anterior nucleus [VA], ventral lateral nucleus

$467[\mathrm{VL}])$.

468 3. Results

$469 \quad 3.1$ Behavioral Measures of Speech Motor Sequence Learning

470 3.1.1. Training Phase. Table 3 shows a summary of the behavioral results for each phase of the

471 experiment and for each group (ANS vs. AWS). Our first set of analyses examined, at the

472 behavioral level, whether participants showed evidence of incremental speech motor sequence

473 learning over the course of the training phase by examining the time course of improvement in

474 each of the three performance measures - mean error rates, utterance durations, and RTs - over

475 the two days of speech motor practice. Each performance measure was averaged within each

476 group, training block, condition, and participant. Again, duration and RT measures were only 


\section{NEURAL CORRELATES OF SPEECH MOTOR SEQUENCE LEARNING}

477 analyzed for utterances that were perceptually coded as having been properly executed (see above)

478 on each day. To visualize the running estimate of the learning trajectories across participants,

479 Figure 1 plots the time course of improvement of each performance measure as a function of group,

480 training block, and condition. Separate analyses of variance (ANOVAs) were performed on the

481 mean sequencing error rates, durations, and RTs with training block (1-8) and condition (native

482 vs. non-native) as within-subjects factors and group (ANS vs. AWS) as a between-subjects factor.

483 In these and all subsequent ANOVAs, Greenhouse-Geisser corrections were applied when

484 appropriate and partial eta-squared effect sizes were calculated for all main effects and 485 interactions. Post-hoc pairwise comparisons were reported as significant at the .05 level.

The ANOVA performed on mean error rates (shown in Figure 2A) revealed a significant main effect of group $\left[F(1,29)=5.036, p=.033, \eta^{2}=.148\right]$, such that AWS [mean $(M)=32.7$;

489 standard deviation $(S D)=13.6]$ produced more sequencing errors than ANS $[M=23.0 ; S D=10.3]$

490 in general. There were also highly significant main effects of condition $[F(1,29)=208.822, p$

$\left.491<.001, \eta_{p}^{2}=.878\right]$ and block $\left[F(7,203)=15.985, \mathrm{p}<.001, \eta^{2}{ }_{p}=.355\right]$. There was one significant

492 interaction, the stimulus condition $X$ block interaction $\left[F(7,203)=8.863, p<.001, \eta^{2} p=.234\right]$. All

493 other interactions were not significant $[p>.05$, in all cases]. Simple effects tests on the condition

$494 \quad \mathrm{X}$ block interaction revealed that there was a significant effect of block on both the native sequences $495\left[F(7,210)=2.958, p=0.045, \eta^{2} p=0.090\right]$ and the non-native sequences $[F(7,210)=20.495, p<$ $\left.4960.001, \eta_{p}^{2}=0.406\right]$. However, the effect size was considerably larger in the non-native condition, 497 indicating that sequence learning was greater for novel words with unfamiliar consonant clusters 498 than novel words with familiar consonant clusters during training. This is in keeping with the 


\section{NEURAL CORRELATES OF SPEECH MOTOR SEQUENCE LEARNING}

499 behavioral results reported by Segawa and colleagues $(2015,2019)$ which found that relatively

500 less learning occurred for native compared to nonnative sequences.

The ANOVA performed on mean utterance duration (shown in Figure 1B) revealed a main

502 effect of group $\left[\mathrm{F}(1,29)=8.963, p=.006, \eta^{2} p=.236\right]$, such that ANS produced shorter utterances $[M$

$503=.59 ; S D=.05]$ than AWS $[M=.69 ; S D=.10]$. There was also a significant effect of stimulus

504 condition $\left[\mathrm{F}(1,7)=34.318, p<.001, \eta^{2}{ }_{p}=.542\right]$, such that the native sequences $[M=.61 ; S D=.09]$

505 were uttered faster than the non-native sequences $[M=.67 ; S D=.10]$, as well as a condition $\mathrm{X}$

506 block interaction $\left[\mathrm{F}(7,203)=2.066, p=.049, \eta^{2}=.066\right]$. All other interactions were not significant

$507[p>.05$, in all cases]. Consistent with our expectations based on our prior studies, simple effects

508 tests on the condition $\mathrm{X}$ block interaction revealed that there was a significant effect of block on

509 the non-native sequences $\left[F(7,210)=3.340, p=0.002, \eta^{2}{ }_{p}=0.100\right]$ but not on the native sequences

$510\left[F(7,210)=1.038, p=0.382, \eta^{2} p=0.033\right]$. Thus, participants only got reliably faster at executing

511 novel words during training if they contained unfamiliar consonant clusters, likely because they

512 were near ceiling performance for novel words using native clusters.

513 The ANOVA performed on mean RT scores (shown in Figure 1C) revealed a highly

514 significant effect for block $\left[F(7,203)=16.742, p<.001, \eta^{2}{ }_{p}=.366\right]$, such that participants got

515 faster at initiating their utterances during the training sessions. The effect of group, condition, and

516 all other interactions were not significant $[p>.05$ in all cases $]$.

In an additional analysis, we examined the mean disfluency rates for the AWS group only.

518 These are shown in Figure 2 averaged across each sequence type (native vs. non-native) and block

519 (1-8). An ANOVA on these scores - sequence type X block - showed no significant main effects

520 or interaction $[p>.05$ in all cases], indicating that stuttering speakers were equally disfluent for

521 both sequence types throughout training. 


\section{NEURAL CORRELATES OF SPEECH MOTOR SEQUENCE LEARNING}

\subsubsection{Test Phase.}

Our second set of analyses examined the same set of behavioral measures of sequence generation while participants were inside the MRI scanner during the test phase. Each performance measure was averaged within each group, testing block, condition, and participant. Duration and RT measures were again only analyzed for utterances coded as properly sequenced productions (see above) on each day. Table 4 shows the mean error rates during the test phase as a function of error subtype and sequence type. Figure 3 shows the mean error rates, utterance duration, and RT scores as a function of group, test block, and condition. Separate analyses of variance (ANOVAs) were performed on the mean error rates, utterance durations, and RTs with group (AWS vs. ANS) as a between-subjects factor, and test block (1-8) and experimental condition (practiced native vs. practiced non-native vs. novel non-native) as within-subjects factors. -- [Insert Table 4 about here] --

The ANOVA performed on mean error rates (shown in Figure 3A) revealed significant main effects of block $\left[F(7,196)=5.291, p<.001, \eta^{2}=.159\right]$ and condition $[F(2,56)=91.164, p$ $\left.<.001, \eta^{2}=.765\right]$. There was no significant main effect of group $\left[F(1,28)=1.371, p=.251, \eta^{2}=\right.$ $.092]$ or interaction effects $[p>.05$ in all cases]. Post-hoc $t$-test comparisons performed on the main effect of condition indicated that, regardless of group, the mean error rates for the practiced native syllables $[M=4.0 ; S D=7.2]$ were significantly lower than the practiced non-native syllables $[M=34.8 ; S D=20.2 ; t(30)=-9.357, p<.001$, Cohen's $d=2.03]$ and novel non-native syllables $[M=44.7 ; S D=21.0 ; t(30)=-10.978, p<.001, d=2.59]$, and the mean error rates for the practiced non-native syllables were lower than the novel non-native syllables $[t(30)=-4.653$, $p<.001, d=.48]$ 


\section{NEURAL CORRELATES OF SPEECH MOTOR SEQUENCE LEARNING}

Measures of mean utterance duration (shown in Figure 3B) patterned similarly. The ANOVA performed on mean utterance durations indicated that there also significant main effects of block $\left[F(7,203)=2.638, p=.033, \eta^{2}=.083\right]$ and condition $\left[F(2,58)=40.733, p<.001, \eta^{2}=\right.$ .584]. Again, there was no significant main effect of group $\left[F(1,29)=.112, p=.740, \eta^{2}=.004\right]$ or interaction effects $[p>.05$ in all cases]. Post-hoc $t$-test comparisons performed on the main

549 effect of condition indicated that, regardless of group, participants were faster at executing the practiced native syllables $[M=.67 ; S D=.15]$ than the practiced non-native syllables $[M=.74$; $S D=.16 ; t(30)=-6.797, p<.001, d=.45]$ and novel non-native syllables $[M=.77 ; S D=.19$;

$t(30)=-7.454, p<.001, d=.58]$. In addition, participants were faster at executing the practiced non-native syllables than the novel non-native syllables $[t(30)=-3.044, p=.005, d=.17]$. of block $\left[F(7,203)=3.114, p=.039, \eta^{2}=.097\right]$ and group $\left[F(1,29)=5.926, p=.021, \eta^{2} p=\right.$ $.170]$, such that AWS took longer to initiate their utterances $[M=.71 ; S D=.36]$ than ANS $[M=$ $.48 ; S D=.16]$. The condition $\times$ group interaction effect also reached statistical significance $[F(2$, 58) $\left.=4.973, p=.012, \eta^{2}{ }_{p}=.146\right]$. Simple effects tests on the condition $\mathrm{X}$ group interaction indicated that the effect of condition approached significance in $\operatorname{AWS}[F(2,30)=3.555, p=.053$, $\left.561 \eta_{p}^{2}=.369\right]$, but not in ANS $\left[F(2,28)=.540, p=.589, \eta_{p}^{2}=.037\right]$. All other interactions were not significant $[p>.05$ in all cases $]$.

Finally, we again examined mean disfluency rates for the AWS group while in the scanner.

564 These scores are shown in Figure 4. The ANOVA performed on these scores - sequence type $X$ 565 block - revealed a highly significant main effect of condition $\left[F(2,30)=9.959, p=<.001, \eta^{2}{ }_{p}=\right.$ $566.399]$. There was no significant main effect of block or interaction effect [ $p>.05$ in both cases]. 


\section{NEURAL CORRELATES OF SPEECH MOTOR SEQUENCE LEARNING}

567 Post-hoc $t$-test comparisons performed on the main effect of condition indicated that AWS were

568 less disfluent when executing the practiced native syllables $[M=4.6 ; S D=11.8]$ compared to the

569 practiced non-native syllables $[M=7.5 ; S D=15.6 ; t(15)=-2.442, p=.027, d=.32]$ and novel

570 non-native syllables $[M=9.8 ; S D=19.4 ; t(15)=-3.476, p=.003, d=.49]$. As well, AWS were

571 less disfluent when producing the practiced non-native than the novel non-native syllables $[t(15)$

$572=-2.307, p=.036, d=.19]$.

573 In summary, we obtained similar results as in our prior studies on speech motor sequence

574 learning with neurotypical speakers (Segawa et al., 2015, 2019): Participants - ANS and AWS

575 alike - showed incremental improvements in performance speed and accuracy, especially for the

576 non-native ones, with repetition and practice during training and these gains were maintained

577 overnight in-between experimental sessions. Moreover, the data indicated that, after two days of

578 extended speech motor practice, speakers from both groups showed further performance gains in

579 accuracy (reduced error rate) for the practiced non-native sequences throughout the test phase

580 (Figure 3A). Although both groups showed comparable gains, the AWS produced the sequences

581 slower and/or less accurately prior to and after training. Overall, these findings provide evidence

582 at the behavioral level that AWS do not show deficits in the acquisition or retention of new speech

583 motor sequences. Finally, RT scores were notably higher during test (inside the scanner) than

584 during training (outside of the scanner), especially for the AWS group. Given that the experimental

585 paradigm during the test phase was identical to that used during the training phase, this difference

586 in initiation speed likely reflects differences in performance anxiety outside versus inside the

587 scanner.

588 3.2. Neural correlates of speech motor sequence learning

589 3.2.1 FMRI analysis 


\section{NEURAL CORRELATES OF SPEECH MOTOR SEQUENCE LEARNING}

590 Before performing direct group comparisons, we first assessed differences between the condition-

591 specific brain activations using the pooled results (ANS and AWS combined). Figure 5A and Table

5925 show the brain regions that were significantly more active during the execution of novel non-

593 native than practiced non-native syllables (TFCE cluster-level $\left.\mathrm{p}_{\mathrm{FWE}}<.05\right)^{3}$ aggregated across both

594 experimental groups. The surface/vertex-based analyses revealed that the production of novel non-

595 native syllables resulted in greater BOLD response in pre-SMA, aINS, aFO, pFO, and IPS

596 bilaterally. In the left hemisphere, additional cortical clusters were noted with peaks in IFS, vPMC,

597 pIFG, midPMC, vMC, midMC, STG, and inferior temporal occipital (ITO) region. The

598 volume/voxel-based analysis found no statistically significant differences in subcortical activity.

599 We supplemented this voxel-based analysis with a hypothesis-based subcortical ROI analysis

600 based on the results reported in Segawa et al. (2015) for this contrast. Specifically, we tested for

601 differences in activation in left globus pallidus internal (GPi) and external (GPe). Results from this

602 analysis demonstrated that left GPi was more activate during production of the novel non-native

603 than the practiced non-native words $[t(30)=2.22, p-u n c=.034, r=.375]$, in effect replicating the

604 finding of Segawa et al. (2015) for the same contrast. No significant groups effects (ANS vs. AWS)

605 were found for any cortical or subcortical region in either hemisphere for the novel non-native -

606 practiced non-native contrast; nevertheless, we present the results (albeit non-significant) for the

607 two groups separately in Figures 5B.

608

Figure $6 \mathrm{~A}$ and Table 6 show the brain regions that were significantly more active during

609 the execution of practiced non-native than practiced native syllables (TFCE cluster-level $\mathrm{p}_{\mathrm{FWE}}<$

610 .05). The surface/vertex-based analyses revealed that the production of practiced non-native words

611 resulted in greater BOLD response in pSTG and OC bilaterally. The increased activity in OC likely

\footnotetext{
${ }^{3}$ For each of the two speech contrast conditions, we report the results from one-sided (positive only) tests.
} 


\section{NEURAL CORRELATES OF SPEECH MOTOR SEQUENCE LEARNING}

612 reflects greater attention and/or depth of processing while reading the non-native text stimuli,

613 rather than differences in speech production. In the left hemisphere, additional cortical clusters

614 were noted with peaks in pre-SMA, aINS, FO, pIFG, vPMC, midPMC, aSTG, vSC, and IPS. The

615 volume/voxel-based analysis found increased activation in the right cerebellum (lobule XIII)

616 during the practiced non-native than the practiced native condition. A hypothesis-based

617 subcortical ROI analysis based on the results reported in Segawa et al. (2015) for this contrast was

618 then performed. Specifically, we tested for differences in activation in right cerebellum (lobule

619 VI). Results from this analysis demonstrated that this region was more activate during production

620 of the practiced non-native than the practiced native words $[t(30)=2.61, p-F D R=.014, r=.430]$,

621 again replicating findings from Segawa et al. (2015). As in the other contrast, no region in either

622 hemisphere was found to be significantly more active for this contrast in either group (ANS vs.

623 AWS) at the cortical or subcortical levels, further bolstering the view that speech motor learning

624 mechanisms are unimpaired in stuttering; again, we present the results (albeit non-significant) for

625 the two groups separately in Figures 6B.

626 Although no significant groups effects were found, several interesting trends emerged that

627 are worth noting and speculating about. First, brain areas linked to speech premotor planning

628 (vPMC, mid-PMC, FO, aINS) showed a non-significant trend toward greater activation in ANS

629 during novel sequence production, suggesting that neurotypical speakers may show greater

630 learning of the motor "chunks" for the trained sequences than AWS. Second, brain areas involved

631 in auditory processing (pSTG) showed a non-significant trend toward greater activation in AWS

632 during novel sequence production, suggesting that, following motor practice, stuttering speakers

633 may still be relying more on auditory feedback to guide them toward the intended production 


\section{NEURAL CORRELATES OF SPEECH MOTOR SEQUENCE LEARNING}

634 targets compared to ANS. Critically, however, there were no brain areas activated in AWS that

635 fell outside the network of areas identified in ANS and in the previous study (Segawa et al., 2015).

$636 \quad 3.3$ Brain-behavior correlation analysis

637 The correlation tests between behavioral measures of learning success and mean activation in the 63812 hypothesis-based ROIs from Segawa et al. (2015) (listed in Table 2) for the novel non-native639 practiced non-native contrast revealed no group differences at either the cortical or subcortical 640 levels. In the analyses performed on the pooled data (ANS and AWS combined), we found that 641 learning success, as measured by the participant-normalized difference in mean error rates between 642 the novel non-native and practiced non-native words, was positively correlated with the mean 643 activation in both the left $[t(29)=2.22, p=.034, r=.381]$ and right aINS $[t(29)=2.38, p=.024$, $644 r=.404]$, such that processing in these areas increased most with learning in participants who 645 showed the greatest performance improvements. These results provide support for the hypothesis 646 that these premotor areas are involved in speech motor sequence learning. No significant 647 correlations emerged between the participant-normalized differences in mean utterance duration 648 (between the novel non-native and practiced non-native words) and the mean activation in any of 649 the ROIs (in all cases, $p_{\text {unc }}>.05$ ).

Finally, within the AWS group, we assessed whether stuttering severity (as indexed by SSI

652 scores and mean disfluency rates inside the scanner during the test phase) was correlated with 653 activation in 8 hypothesis-based ROIs within the cortico-BG loops (listed in Table 2) for the novel 654 non-native - practiced non-native contrast. Correlation tests revealed that mean disfluency rates 655 inside the scanner were negatively correlated with mean activation in left GPe $[t(14)=-2.88, p$ $656 F D R=.047, r=-0.609]$ and left caudate $[t(14)=-2.74, p-F D R=.047, r=-.591]$ (i.e., the more 


\section{NEURAL CORRELATES OF SPEECH MOTOR SEQUENCE LEARNING}

657 disfluent participants were during the test phase, the less BOLD activity change was observed in

658 these basal ganglia sites for this contrast). Although this result is correlational and therefore a

659 causal relationship cannot be firmly established, it is nonetheless compatible with the idea that

660 speakers are more likely to stutter when the left GPe and left caudate are more hypoactive. In

661 contrast, no statistically significant correlations were found between SSI scores and activation in

662 any of the 8 ROIs. The latter result is perhaps unsurprising given that the SSI does not measure

663 disfluency during non-native sequence production and its affiliated scores were not based on

664 speech uttered inside the scanner.

-- [Insert Figure 8 about here] --

In summary, we found that, across both ANS and AWS, greater activity was observed

667 during the production of novel sequences in brain regions previously associated with learning and

668 maintaining speech motor programs, including lateral premotor cortex, FO, aINS, posterior

669 superior temporal cortex, and right cerebellum (Segawa et al., 2015; Guenther, 2016). Measures

670 of learning success correlated positively with activity in the left and right aINS, suggesting that

671 this brain area plays an important role in speech motor learning. In AWS, measures of in-scanner

672 disfluency rates were negatively correlated with activity in left-lateralized basal ganglia structures.

673 Overall, these findings provide evidence that the neurobiological correlates of speech motor

674 sequence learning do not differ across ANS and AWS.

\section{4. Discussion}

676 The goal of the current study was to investigate the behavioral and neural correlates of speech

677 motor sequence learning in both stuttering and neurotypical adult speakers. Using a cued sequence

678 production task, we examined changes in the performance of pseudowords containing non-native

679 consonant clusters over time as a function of speech motor practice. At the behavioral level, we 


\section{NEURAL CORRELATES OF SPEECH MOTOR SEQUENCE LEARNING}

680 found that, although AWS were less accurate and/or slower compared to ANS at producing words

681 involving non-native consonant clusters regardless of whether the words were practiced, they

682 showed statistically comparable improvements in these measures with practice to those seen in

683 ANS. At the neural level, we found that, across both groups and both speech contrasts (novel non-

684 native - practiced non-native and practiced non-native - practiced native), practice producing

685 words with non-native clusters led to significant decreases in BOLD activity in brain areas

686 implicated in verbal working memory and speech motor planning (including left preSMA, FO,

687 aINS, and bilateral IPS), suggesting that processing load and articulatory effort decreases as the

688 nervous system forms regularized motor programs (or "chunks") for coordinated sequences of

689 vocal tract gestures. Critically, there were no reliable group differences in either contrast map in

690 any areas involved in the cortico-BG loops (or otherwise). Furthermore, the degree of motor

691 performance gains in accuracy were correlated positively with activity in the left and right aINS.

692 Collectively, these findings suggest that cortico-BG-mediated mechanisms involved in learning

693 novel phoneme sequences are not impaired in AWS; instead, the impairment is primarily in the

694 motor execution of speech sequences, novel and learned alike.

695 As in our prior study (Segawa et al., 2015), we also observed pre- to post-training BOLD

696 activation changes in cortical areas that extend beyond those implicated in verbal working memory

697 and speech motor planning processes. Specifically, we found greater activity in areas related to 698 auditory processes (left PT, aSTG, and pSTG) during production of the novel non-native sequences

699 relative to the practiced non-native sequences (Figure 5). These auditory regions are thought to be

700 involved in guiding speech movements based on self-generated auditory feedback (Hickok, 2012;

701 Guenther, 2016). During speech production, activity in this area has been reported to be greater 702 when there is a mismatch between predicted and actual auditory feedback (Tourville, Reilly, \& 


\section{NEURAL CORRELATES OF SPEECH MOTOR SEQUENCE LEARNING}

703 Guenther, 2008). Several existing theoretical models of speech production (e.g., Hickok, 2012;

704 Guenther, 2016) propose that error signals arising from these regions are used to fine-tune speech

705 motor programs over the course of repeated production attempts. Thus, learning is thought to rely

706 on the transmission of these auditory error signals to frontal regions involved in motor planning

707 and execution.

708 We also noted greater activity in cortical areas related to orthographic processing (left ITO)

709 during production of the novel non-native sequences relative to the practiced non-native sequences

710 (Figure 5). Prior neuroimaging studies suggest that ITO is a higher-level visual processing area

711 involved in identifying letters and words from lower-level shape images (see, e.g., Pugh, Mencl,

712 Jenner, Katz, Frost, Lee, et al., 2001) and is therefore highly likely to be related to viewing and

713 decoding the pseudowords that our participants were instructed to produce, rather than speech

714 motor control. In contrast, production of the practiced non-native sequences did not produce

715 significantly greater activity compared with the practiced native sequences in ITO (Figure 6). This

716 difference between the condition-specific brain activations suggests that pseudowords containing

717 unfamiliar phonotactic sequences are more difficult to process prior to reading and repetition

718 practice (cf. Pugh, Frost, Sandak, Landi, Rueckl, Constable, Seidenberg, Fulbright, Katz, \& Mencl, 719 2008).

720 The finding that the right cerebellum (which is structurally and functionally connected with

721 left cortical areas) was recruited to a greater extent across both groups during the production of the

722 practiced non-native than the practiced native sequences (shown in Figure 6) was also observed

723 by Segawa et al. (2015) in ANS, and corroborates other reports with neurotypical participants that

724 the cerebellum plays an important role in motor sequence learning and fine motor control (e.g.,

725 Doyon, Song, Karni, Lalonde, Adama, \& Ugerleider, 2002; Ackermann, 2008; Guenther, 2016, 


\section{NEURAL CORRELATES OF SPEECH MOTOR SEQUENCE LEARNING}

726 chapter 2). For example, Bohland and Guenther (2006) found that different regions of the

727 cerebellum showed differential sensitivity to syllable complexity (e.g., "stra vs. ta") and serial

728 complexity (e.g., "da-da-da” vs. "da-ru-ti”) in ANS, suggesting involvement in speech sequencing.

729 That said, it is somewhat surprising that we did not find significant differences in cerebellar activity

730 in the novel non-native - practiced non-native contrast. However, this comparison possessed less

731 statistical power because of more removed error trials.

732 An alternative, but not mutually exclusive, interpretation to the cortical activity differences

733 we found for both speech contrasts is that these differences result from the fact that the novel non-

734 native and practiced non-native sequences are more difficult to vocally imitate than the practiced

735 native sequences. Two sources of evidence support this conjecture. First, prior neuroimaging

736 studies (e.g., Irwin, Frost, Mencl, Chen \& Fowler, 2011) have reported that imitation of auditory

737 speech produces additional significant activations in left IFG and aINS when compared to a

738 passive listening condition. Second, current computational models, such as the Directions Into

739 Velocities of the Articulators (DIVA) model (Guenther, 2016), propose that speech motor learning

740 is driven by initial mismatches between newly acquired sound targets and one's own production

741 attempts as represented in the auditory cortex. Auditory error signals are then transformed into

742 corrective motor commands, and these corrective commands alter the feedforward commands for

743 the next production attempt. As the feedforward commands improve, fewer error signals are

744 generated and thus the contribution of the feedback control system gradually diminishes. The

745 DIVA model thus predicts decreases in BOLD activation in both motor planning and auditory

746 cortical areas as a consequence of imitation-based learning. In this view, the nervous system begins

747 to form speech motor programs via an imitation-based learning mechanism, which may account

748 for the increased activity in motor and auditory areas for the novel non-native compared with the 


\section{NEURAL CORRELATES OF SPEECH MOTOR SEQUENCE LEARNING}

practiced non-native sequences, and also for the practiced non-native compared with the practiced native sequences.

A number of other neuroimaging studies, compiled and discussed in a meta-analysis by Belyk and colleagues (Belyk, Kraft, \& Brown, 2015), have reported that AWS tend to display higher activity in a number of right-hemisphere regions during fluently produced native speech when compared to ANS. Some researchers have proposed that this right-hemisphere cortical hyperactivity arises from impaired left-hemisphere function (see, e.g., Fox et al., 1996; Belyk et al., 2015; Guenther, 2016; Neef, Anwander, Butfering, Schmidt-Samoa, Friederici, Paulus, \& Sommer, 2017; cf. Connalley et al., 2018). In view of these results, it is perhaps surprising that no clusters emerged in the right hemisphere for AWS in the current study. However, it is possible that right-hemisphere hyperactivity in AWS may not occur in all speaking situations. In particular, the current speaking task likely required more attention and articulatory effort than those in prior studies since the participants were required to produce non-native sequences of segments, which is known to be difficult (e.g., Davidson, 2006, 2010). In addition, there may be methodological issues that concern the difference between the experimental procedures we used to test for a functional activation difference between ANS and AWS and the ones used by other research groups as the existing literature on stuttering encompasses a diverse set of sampling protocols and motor tasks.

In the current study, it was further demonstrated, in AWS, that in-scanner mean disfluency covaried with BOLD responses in left-lateralized basal ganglia sites (GPe and left caudate), such that the more disfluent participants were during the test phase, the less BOLD activity change was observed in these basal ganglia sites for the novel non-native - practiced non-native contrast. This negative correlation does not establish a causal relationship; rather it establishes an associative 


\section{NEURAL CORRELATES OF SPEECH MOTOR SEQUENCE LEARNING}

772 link. Although this relationship is broadly consistent with the long-standing view that stuttering

773 reflects a malfunction within the cortico-BG loops (e.g., Fox et al., 1996; Alm, 2004; Giraud et

774 al., 2007; Watkins et al., 2008; Kell, Neumann, von Kriegstein, Posenenske, von Gudenberg,

775 Euler, \& Giraud, 2009; Lu, Chen, Ning, Ding, Guo, Peng, Yang, Li, \& Lin, 2010; Connally et al.,

776 2018; Chang \& Guenther, 2020), it would appear to be at odds with other neuroimaging studies

777 (albeit that did not use a sparse-sampling method) which have reported positive relationships

778 between basal ganglia activity and stuttering severity (e.g., Giraud et al., 2008; see also, Metzger

779 et al., 2018, for a similar finding based on manual motor responses in AWS). Differences between

780 the findings of the current study and these prior studies suggest that the brain mechanisms

781 underlying general stuttering traits and transient disfluent states are dissociable (for further

782 discussion, see Connally et al., 2018).

783 At first glance, the lack of a robust learning difference between the ANS and AWS groups

784 may appear to be at odds with other behavioral studies reporting motor learning deficits in AWS

785 (Ludlow, Siren, \& Zikria, 1997; Smits-Bandstra, De Nil, \& Saint-Cyr, 2006; Namasivayam \& van

786 Lieshout, 2008). Several important differences between those studies and ours might account for

787 the divergent results. First, our study measured sequence and cluster learning using perception-

788 based segmental transcription and simple acoustic measures. However, this approach required

789 transcribers to make categorical decisions regarding the segments that speakers produce, and thus

790 did not permit quantitative analysis of the presence or magnitude of various gestures, or how

791 speakers continually updated their implementation of novel gestural scores. In contrast, other

792 motor learning studies (e.g., Namasivayam \& van Lieshout, 2008) used kinematic measures to

793 compare more subtle aspects of speech articulation between ANS and AWS. Thus, subtle

794 differences in the articulatory correlates of speech motor learning may exist between ANS and 


\section{NEURAL CORRELATES OF SPEECH MOTOR SEQUENCE LEARNING}

795 AWS. Ongoing studies based on the current design that use electromagnetic articulography will

796 determine whether AWS and ANS differ in their ability to learn to execute and coordinate inter-

797 articulator movements for novel speech sequences.

798 Second, our study focused on the learning of new phoneme sequences within a single

799 syllable, with which the participants had no prior experience since they violated English

800 phonotactic constraints. This task required learning of new articulatory sequences at a motoric

801 level. In contrast, other studies in the literature on speech motor sequence learning in AWS have

802 involved production of more extended, multi-syllabic sequences that conform to English

803 phonotactics (e.g., Namasivayam \& van Lieshout, 2008). However, there is evidence that multi-

804 syllabic sequences places higher demands on phonological working memory mechanisms than

805 motor programming mechanisms. For example, in another fMRI study, McGettigan, Warren,

806 Eisner, Marshall, Shanmugalingam, and Scott (2010) found that activity in planum temporale, a

807 region implicated in auditory working memory, increased as the number of syllables in either a

808 non-word repetition or passive listening task increased, whereas activity in left SMA, a region

809 implicated in speech motor programming, increased as the number of consonant clusters increased.

810 In this light, the increased error rates reported in other studies with AWS might be interpreted

811 instead as reflecting differences in phonological working memory mechanisms, rather than motor

812 learning or implementation mechanisms (see also, Yang, Jia, Fox, Siok, \& Tan, 2019). Yet others

813 have reported some differences between ANS and AWS in their degree of motor adaptation

814 following an experimental perturbation to their online sensory feedback (e.g., Daliri, Wieland, Cai,

815 Guenther, \& Chang, 2018), suggesting that mechanisms involved in updating existing motor

816 programs may still be impaired in stuttering. 


\section{NEURAL CORRELATES OF SPEECH MOTOR SEQUENCE LEARNING}

It is also important to note that the present fMRI measures focused on the outcome of

818 speech motor sequence learning, not the online process of learning. However, previous research,

819 spanning a wide range of learning tasks and paradigms, has shown that participants may have

820 similar learning outcomes despite very different learning trajectories (see Karuza, Emberson, \&

821 Aslin, 2014, for a review). Thus, despite showing comparable BOLD activity patterns at test, ANS

822 and AWS may not show evidence of the same, qualitative neural changes during the course of

823 speech motor sequence training. It would therefore be of interest to conduct further fMRI studies,

824 based on the current design, that perform BOLD contrast analyses throughout both the training

825 and test phases.

826 Finally, the current study focused primarily on the mechanisms of speech motor learning

827 and control, from the phonetic encoding stage down to the motor commands to the speech

828 articulators. Specifically, we examined how ANS and AWS learn to transform discrete

829 "phonological chunks" (speech sounds, which can be phonemes, syllables, or words) into a set of

830 articulator movements that achieves the intended auditory "target." We employed monosyllabic,

831 pseudoword stimuli to provide a "purer" measure of the speech motor system, as this would limit

832 the recruitment of higher-level cognitive and linguistic processing strategies to assist task

833 performance (see also, McGettigan et al., 2010). However, any viable theory of speech production

834 learning will ultimately have to explicate how these phonological chunks are integrated with

835 prosodic structures, as well as their relation to syntactic/semantic planning processes. Future

836 studies will aim to develop predictions from the highly controlled laboratory experiments

837 presented here and elsewhere (e.g., Segawa et al., 2015, 2019) that may be tested when speakers

838 are instructed to produce novel speech sequences in a more natural context. 
NEURAL CORRELATES OF SPEECH MOTOR SEQUENCE LEARNING

\section{References}

Ackermann, H. (2008). Cerebellar contributions to speech production and speech perception: psycholinguistic and neurobiological perspectives. Trends in Neurosciences, 31, 265-272.

Alexander, G.E., DeLong, M.R., \& Strick, P.L. (1986). Parallel organization of functionally segregated circuits linking basal ganglia and cortex. Annual Review of Neuroscience, 9, $357-381$.

Alm, P. A. (2004). Stuttering and the basal ganglia circuits: a critical review of possible relations. Journal of Communication Disorders, 37, 325-369. doi: 10.1016/j.jcomdis.2004.03.001

Anderson, J.L., Hutton, C., Ashburner, J., Turner, R., \& Friston, K. (2001). Modeling geometric deformations in EPI time series. NeuroImage, 13(5), 903-919.

Ashburner, J., \& Friston, K.J. (2005). Unified segmentation. NeuroImage, 26(3), 839-851.

Belin, P., Zatorre, R. J., Hoge, R., Evans, A. C., \& Pike, B. (1999). Event-related fMRI of the auditory cortex. NeuroImage, 10, 417-429.

Belyk, M., Kraft, S. J., \& Brown, S. (2015). Stuttering as a trait or state—an ALE meta-analysis of neuroimaging studies. European Journal of Neuroscience, 41, 275-284.

Berent, I., Steriade, D., Lennertz, T., \& Vaknin, V. (2007). What we know about what we have never heard: Evidence from perceptual illusions. Cognition, 104, 591-630.

Bloodstein, O., and Ratner, N. B. (2008). A handbook on stuttering. Clifton Park, NY: Thomson/Delmar Learning, 552.

Bohland, J.W., Bullock, D., \& Guenther, F.H. (2010). Neural representations and mechanisms for the performance of simple speech sequences. Journal of Cognitive Neuroscience, 22(7), pp. 1504-1529.

Boersma, P., \& Weenink, D. (2020). Praat: doing phonetics by computer [Computer 
NEURAL CORRELATES OF SPEECH MOTOR SEQUENCE LEARNING

program]. Version 6.1.09, retrieved 26 January 2020 from http://www.praat.org/

Bohland, J. W., \& Guenther, F. H. (2006). An fMRI investigation of syllable sequence production. NeuroImage, 32, 821-841.

Boutsen, F. (1993). A comparative study of stress timing of stutterers and nonstutterers. Journal of Fluency Disorders, 20, 145-155.

Brawn, T.P., Fenn, K.M., Nusbaum, H.C., \& Margoliash, D. (2010). Consolidating the effects of waking and sleep on motor-sequence learning. Journal of Neuroscience, 30(42), 1397713982.

Buchwald, A., Calhoun, H., Rimikis, S., Steinberg Lowe, M, Wellner, R., \& Edwards, D. (2019). Using tDCS to facilitate motor learning in speech production: The role of timing. Cortex,

Byrd, D. (1996). Influences on articulatory timing in consonant sequences. Journal of Phonetics,

Cai, S., Tourville, J. A., Beal, D. S., Perkell, J. S., Guenther, F. H., \& Ghosh, S. S. (2014). Diffusion imaging of cerebral white matter in persons who stutter: evidence for network-level canomalies. Frontiers in Human Neuroscience, 8, 18 pages.

Chang, S.-E., Ohde, R.N., \& Conture, E.G. (2002). Coarticulation and formant transition rate in young children who stutter. Journal of Speech, Language, and Hearing Research, 45, 676production. Cognition, 99, 205-235. 


\section{NEURAL CORRELATES OF SPEECH MOTOR SEQUENCE LEARNING}

885 Civier, O., Bullock, D., Max, L., and Guenther, F. H. (2013). Computational modeling of stuttering caused by impairments in a basal ganglia thalamocortical circuit involved in syllable selection and initiation. Brain and Language. 126, 263-278.

888 Connally, E.L., Ward, D., Pliatsikas, C., Finnegan, S., Jenkinson, M., Boyles, R., Watkins, K.E. (2018). Separation of trait and state in stuttering. Human Brain Mapping, 39, 3109-3126.

Coltheart, M. (1981). The MRC Psycholinguistic Database. The Quarterly Journal of Experimental Psychology Section A. 33(4), 497-505.

Craig-McQuaide, A., Akram, H., Zrinzo, L., \& Tripoliti, E. (2014). A review of brain circuitries involved in stuttering. Frontiers in Human Neuroscience, doi: 10.3389/fnhum.2014.00884

Dale, A. M., Fischl, B., \& Sereno, M. I. (1999). Cortical surface- based analysis-I. Segmentation and surface reconstruction. NeuroImage, 9, 179-194.

Daliri, A., Wieland, E. A., Cai, S., Guenther, F. H., \& Chang, S. E. (2018). Auditory-motor

Davidson, L. (2006). Phonology, phonetics, or frequency: Influences on the production of nonnative sequences. Journal of Phonetics, 34, 104-137.

Davidson, L. (2010). Phonetic bases of similarities in cross-language production: Evidence from English and Catalan. Journal of Phonetics, 38, 2, 272-288.

Davidson, L., \& Shaw, J. (2012). Sources of illusion in consonant cluster perception. Journal of

905

906

907 adaptation is reduced in adults who stutter but not in children who stutter. Developmental Science, 21:e12521. Phonetics, 40(2), 234-248.

Diedrichsen, J., Balsters, J. H., Flavell, J., Cussans, E., \& Ramnani, N. (2009). A probabilistic atlas of the human cerebellum. Neuroimage, 15, 39-46.

Doyon, J., Song, A. W., Karni, A., Lalonde, F., Adams, M. M., \& Ungerleider, L. G. (2002). 
NEURAL CORRELATES OF SPEECH MOTOR SEQUENCE LEARNING

Experience-dependent changes in cerebellar contributions to motor sequence learning. Proceedings of the National Academy of Sciences, U.S.A., 99, 1017-1022.

Doyon, J., Gabitov, E., Vahdat, S., Lungu, O., \& Boutin, A. (2018). Current issues related to motor sequence learning in humans. Current Opinion in Behavioral Sciences, 20, 89-97.

Dupoux, E., Kakehi, K., Pallier, Y., Hirose, C., \& Mehler, J. (1999). Epenthetic vowels in Japanese: a perceptual illusion? Journal of Experimental Psychology: Human Perception and Performance, 25(6), 1568-1578.

Dupoux, E., Parlato, E., Frota, S., Hirose, Y., \& Peperkamp, S. (2011). Where do illusory vowels comes from? Journal of Memory and Language, 64(3), 199-210.

Fee, M.S., \& Goldberg, J.H. (2011). A hypothesis for basal ganglia dependent reinforcement

919 learning in the songbird. Journal of Neuroscience, 198, 152-70.

Fenn, K. M., Nusbaum, H. C., \& Margoliash, D. (2003). Consolidation during sleep of perceptual learning of spoken language. Nature, 425, 614-616.

Ferraro, F. R., Balota, D. A., \& Connor, L. T. (1993). Implicit memory and the formation of new associations in nondemented Parkinson's disease individuals and individuals with senile dementia of the Alzheimer type: a serial reaction time (SRT) investigation. Brain and Cognition, 21, 163-180.

Fischl, B., Sereno, M. I., \& Dale, A. M. (1999). Cortical surface-based analysis-II: Inflation, flattening, and a surface-based coordinate system. NeuroImage, 9, 195-207.

Fischl, B., Salat, D. H., Busa, E., Albert, M., Dieterich, M., Haselgrove, C., et al. (2002). Whole brain segmentation: Automated labeling of neuroanatomical structures in the human brain. Neuron, 33, 341-355.

Fox, P. T., Ingham, R. J., Ingham, J. C., Hirsch, T. B., Downs, J. H., Martin, C., Jerabek, P., Glass, 
NEURAL CORRELATES OF SPEECH MOTOR SEQUENCE LEARNING

T., \& Lancaster, J. L. (1996). A PET study of the neural systems of stuttering. Nature, 382(6587), 158-161.

933 Giraud, A.-L., Neumann, K., Bachoud-Levi, A.-C., von Gudenberg, A. W., Euler, H. A.,

934 Lanfermann, H., \& Preibisch, C. (2008). Severity of dysfluency correlates with basal 935 ganglia activity in persistent developmental stuttering. Brain and Language, 104, 190-199.

936 Gracco, V.L., Tremblay, P., Pike, G.B. (2005). Imaging speech production using fMRI.

937 NeuroImage, 26, 294-301.

938 Guenther, F.H. (2016). Neural Control of Speech. Cambridge, MA: MIT Press.

939 Hagler Jr, D. J., Saygin, A. P., \& Sereno, M. I. (2006). Smoothing and cluster thresholding for 940 cortical surface-based group analysis of fMRI data. NeuroImage, 33(4), 1093-1103.

941 Hayasaka, S., \& Nichols, T. E. (2003). Validating cluster size inference: Random field and 942 permutation methods. NeuroImage, 20, 2343-2356.

943 Helmuth, L., Mayr, U., \& Daum, I. (2000). Sequence learning in patients with Parkinson's disease: 944 a comparison between spatial-attention and number-response sequences.

$945 \quad$ Neuropsychologia, 38, 1443-1451.

946 Hickok, G. (2012). Computational neuroanatomy of speech production. Nature Reviews $947 \quad$ Neuroscience, 13, 135-145.

948 Ingham, R. J., Grafton, S. T., Bothe, A. K., \& Ingham, J. C. (2012). Brain activity in adults who 949 stutter: Similarities across speaking tasks and correlations with stuttering frequency and $950 \quad$ speaking rate. Brain and Language, 122(1), 11-24.

951 Irwin, J.R., Forst, S.J., Mencl, E.W., Chen, H., \& Fowler, C.A. (2012). Functional activation for 952 imitation of seen and heard speech. Journal of Neurolinguistics, 24(6), 611-618.

953 Jackson, G. M., Jackson, S. R., Harrison, J., Henderson, L., \& Kennard, C. (1995). Serial reaction 
NEURAL CORRELATES OF SPEECH MOTOR SEQUENCE LEARNING

954

955

956

957

958

959

960

961

962

963

964

965

966

967

968

969

970

971

972

973

974

975

time learning in Parkinson's disease: evidence for a procedural learning deficit. Neuropsychologia, 33, 577-593.

Jezzard, P., \& Balaban, R.S. (1995). Correction for geometric distortion in echo planar images from B0 field variations. Magnetic Resonance in Medicine, 34(1), 65-73.

Karuza, E.A., Emberson, L.L., \& Aslin, R.N. (2014). Combining fMRI and behavioral measures to examine the process of human learning. Neurobiology of Learning and Memory, 109, 193-206.

Kell, C. A., Neumann, K., von Kriegstein, K., Posenenske, C., von Guden- berg, A. W., Euler, H., \& Giraud, A.-L.L. (2009). How the brain repairs stuttering. Brain, 132(10), 2747-2760.

Keuken, M. C., Bazin, P.-L., Crown, L., Hootsmans, J., Laufer, A., Müller-Axt, C., Sier, R., van der Putten, E.J., Schafer, A., Turner, R., Forstmann, B. U. (2014). Quantifying interindividual anatomical variability in the subcortex using 7T structural MRI. Neuroimage, 94, 40-46. https://doi.org/https://doi.org/10.1016/j.neuroimage.2014.03.032

Kotz, S. A., \& Schwartze, M. (2010). Cortical speech processing unplugged: a timely subcorticocortical framework. Trends in Cognitive Science, 14, 392-399.

Lu, C., Chen, C., Ning, N., Ding, G., Guo, T., Peng, D., Yang, Y., Li, K., \& Lin, C. (2010). The neural substrates for atypical planning and execution of word production in stuttering. Experimental Neurology, 221(1), 146-156.

Ludlow, C. L., Siren, K., \& Zikria, M. (1997). Speech production learning in adults with chronic developmental stuttering. In W. Hujstijn, H. F. M. Peters, \& P. H. H. M. Van Lieshout (Eds.), Speech production: Motor control, brain research and fluency disorders (pp. 212229). Amsterdam: Elsevier Science Publishers. 


\section{NEURAL CORRELATES OF SPEECH MOTOR SEQUENCE LEARNING}

976 Max, L, Guenther, F.H., Gracco, V.L., Ghosh, S.S., Wallace, M.E. (2004). Unstable or

977 insufficiently activated internal models and feedback-biased motor control as sources of

978 dysfluency: a theoretical model of stuttering. Contemporary Issues in Communication

$979 \quad$ Science and Disorders, 31, 105-22.

980 Metzger, F.L., Auer, T., Helms, G., Paulus, W., Frahm, J., Sommer, M., \& Neef, N.E. (2018).

981 Shifted dynamic interactions between subcortical nuclei and inferior frontal gyri during 982 response preparation in persistent developmental stuttering. Brain Structure and Function, $983 \quad 223,165-182$.

984 McGettigan, C., Warren, J.E., Eisner, F., Marshall, C.R., Shanmugalingam, P., \& Scott, S.K. (2011). Neural correlates of sublexical processing in phonological working memory. Journal of Cognitive Neuroscience, 23(4), 961-977.

987 Mollaei, F., Shiller, D.M., \& Gracco, V.L. (2013). Sensorimotor adaptation of speech in 988 Parkinson's Disease. Journal of Movement Disorders, 28, 1668-1674.

989 Nakamura, K., Sakai, K., \& Hikosaka, O. (1998). Neuronal activity in medial frontal cortex during $990 \quad$ learning of sequential procedures. Journal of Neurophysiology, 80, 2671-2687.

991 Namasivayam, A. K., \& Van Lieshout, P. H. H. M. (2008). Bite-block perturbation in people who 992 993 Communication Disorders, 41, 372-294.

994 Neef, N. E., Anwander, A., Butfering, C., Schmidt-Samoa, C., Friederici, A. D., Paulus, W., \& 995 Sommer, M. (2017). Structural connectivity of right frontal hyperactive areas scales with 996 stuttering severity. Brain, https://doi.org/10.1093/brain/awx316

997 Niethammer, M., Feigin, A., \& Eidelber, D. (2012). Functional neuroimaging in Parkinson's 998 disease. Cold Spring Harbor Perspectives in Medicine, 2, a009274. 


\section{NEURAL CORRELATES OF SPEECH MOTOR SEQUENCE LEARNING}

999 Nieto-Castañón, A., Ghosh, S.S., Tourville, J.A., Guenther, F.H., 2003. Region of interest based 1000 analysis of functional imaging data. Neuroimage 19, 1303-1316.

1001 Nieto-Castañón, A. (2020). Handbook of functional connectivity magnetic resonance imaging 1002 methods in CONN. Boston, MA: Hilbert, Press.

1003 Pan, S.C., \& Rickard, T.C. (2015). Sleep and motor learning: Is there room for consolidation? Psychological Bulletin, 141(4), 812-834.

1005

1006

1007

1008

1009

1010

1011

1012

1013

1014

1015

1016

1017

1018

1019

1020

1021

Perrachione, T.K., \& Ghosh, S.S. (2013). Optimized design and analysis of sparse-sampling FMRI experiments. Frontiers in Neuroscience. 18(7), doi: 10.3389/fnins.2013.00055

Pitt, M.A. (1998). Phonological processes and the perception of phonotactically illegal consonant clusters. Perception \& Psychophysics, 60(6), 941-951.

Pugh, K. R., Mencl, W. E., Jenner, A. R., Katz, L., Frost, S. J., Lee, J. R., et al. (2001). Neurobiological studies of reading and reading disability. Journal of Communicable Diseases, 34, 479-492.

Pugh, K.R., Forst, S.F., Sandak, R., Landi, N., Rueckl, J.G., Constable, T.R., Seidenberg, M., Fulbright, R., Katz, L., \& Mencl, W.E. (2008). Effects of stimulus difficulty and repetition on printed word identification: an fMRI comparison of non-impaired and reading disabled adolescent cohorts. Journal of Cognitive Neuroscience, 20(7), 1146-1160.

Rauschecker, A. M., Pringle, A., \& Watkins, K. E. (2008). Changes in neural activity associated with learning to articulate novel auditory pseudowords by covert repetition. Human Brain Mapping, 29, 1231-1242.

Riley, G.D. (2009). Stuttering severity instrument for children and adults (SSI-4) 4th ed. Pro-Ed, Inc; Austin, TX.

Robb, M., \& Blomgren, M. (1997). Analysis of F2 transitions in the speech of stutterers and 


\section{NEURAL CORRELATES OF SPEECH MOTOR SEQUENCE LEARNING}

1022

1023

1024

1025

1026

1027

1028

1029

1030

1031

1032

1033

1034

1035

1036

1037

1038

1039

1040

1041

1042

1043

1044

nonstutterers. Journal of Fluency Disorders, 22, 1-16.

Robb, M., Blomgren, M., \& Chen, Y. (1998). Formant frequency fluctuation in stuttering and nonstuttering adults. Journal of Fluency Disorders, 23, 73-84.

Rong, F., Isenberg, A.L., Sun, E., \& Hickok, G. (2018). The neuroanatomy of speech sequencing at the syllable level. PLOS ONE, 13(10): e0196381.

Rottschy, C., Langner, R., Dogan, I., Reetz, K., Laird, A. R., Schulz, J. B., \& Eickhoff, S. B. (2012). Modelling neural correlates of working memory: A coordinate-based metaanalysis. NeuroImage, 60, 830-846.

Sapir, S. (2014). Multiple factors are involved in the dysarthria associated with Parkinson's disease: a review with implications for clinical practice and research. Journal of Speech and Hearing Research, 57, 1330-1343.

Segawa, J., Tourville, J.A., Beal, D.S., \& Guenther, F.H. (2015). The neural correlates of speech motor sequence learning. Journal of Cognitive Neuroscience, 27(4), 819-831.

Segawa, J., Masapollo, M., Tong, M., Smith, D.J., \& Guenther, F.H. (2019). Chunking of phonological units in speech sequencing. Brain and Language, 104636.

Shima, K., \& Tanji, J. (2000). Neuronal activity in the supplementary and presupplementary motor areas for temporal organization of multiple movements. Journal of Neurophysiology, 84, 2148-2160.

Skodda, S. (2012). Effect of deep brain stimulation on speech performance in Parkinson's disease. Parkinson's Disease, 2012, 850596.

Smith, S. M., \& Nichols, T. E. (2009). Threshold-free cluster enhancement: addressing problems of smoothing, threshold dependence and localisation in cluster inference. Neuroimage, 44(1), 83-98. 


\section{NEURAL CORRELATES OF SPEECH MOTOR SEQUENCE LEARNING}

1045 Smits-Bandstra, S., De Nil, L. F., \& Rochon, E. (2006). The transition to increased automaticity

1046 during finger sequence learning in adult males who stutter. Journal of Fluency Disorders,

$1047 \quad 31,22-42$.

1048 Smits-Bandstra, S., \& Gracco, V. (2013). Verbal implicit sequence learning in persons who stutter 1049 and persons with Parkinson's disease. Journal of Motor Behavior, 45, 381-393.

1050 Sternberg, S., Monsell, S., Knoll, R. L., \& Wright, C. E. (1978). The latency and duration of rapid 1051 movement sequences: Comparisons of speech and typing. In G. E. Stelmach (Ed.), 1052 Information processing in motor control and learning (pp. 117-152). New York: $1053 \quad$ Academic Press.

1054 Tourville, J.A. \& Guenther, F.H. (2003). A cortical and cerebellar parcellation system for speech 1055 studies. Boston University Technical Report CAS/CNS-03-022. Boston, MA: Boston 1056 University.

1064 Tourville, J. A., Reilly, K. J., \& Guenther, F. H. (2008). Neural mechanisms underlying auditory 1065 feedback control of speech. Neuroimage, 39, 1429-1443.

1066 Toyomura, A., Fujii, T., Kuriki, S. (2015). Effect of an 8-week practice of externally triggered 1067 1068 speech on basal ganglia activity of stuttering and fluent speakers. NeuroImage, 109, 458-

1069 Vahdat, S., Fogel, S., Benali, H., \& Doyon, J. (2017). Network-wide reorganization of procedural 1070 memory during NREM sleep revealed by fMRI. Elife, 11, e24987.

1071 Watkins, K.E., Smith, S.M., Davis, S., \& Howell, P. (2008). Structural and functional 1072 abnormalities of the motor system in developmental stuttering. Brain, 131, 50-59.

1073 Whitfield-Gabrieli, S., \& Nieto-Castanon, A. (2012). CONN: a functional connectivity toolbox for 1074 correlated and anticorrelated brain networks. Brain connectivity, 2(3), 125-141 


\section{NEURAL CORRELATES OF SPEECH MOTOR SEQUENCE LEARNING}

1075 Wiener, N. (1949). Extrapolation, interpolation, and smoothing of stationary time series.

1076 Cambridge, MA: MIT Press.

1077 Yang, Y., Jia, F., Fox, P.T., Siok, W., \& Tan, L.H. (2019). Abnormal neural response to

1078 phonological working memory demands in persistent developmental stuttering. Human

1079 Brain Mapping, 40(1), 214-225.

1080 Yaruss, J.S. \& Conture, E.G. (1993). F2 transitions during sound/syllable repetitions of children

1081 who stutter and predictions of stuttering chronicity. Journal of Speech, Language, and

1082 Hearing Research, 36, 883-896. 
NEURAL CORRELATES OF SPEECH MOTOR SEQUENCE LEARNING

\section{Figure captions}

1084 Figure 1: Time course of improvement of behavioral performance measures during training 1085 (outside the scanner) for the practiced native and practiced non-native sequences as a function of 1086 training block (1-8) and group (ANS vs. AWS). (A) Mean percentage of errors of each sequence

1087 type; (B) Mean durations of the properly executed utterances of each sequence type; (C) Mean 1088 reaction times of the properly executed utterances of each sequence type. The shaded area 1089 represents the standard error of the mean.

1090 Figure 2: Mean percentage of disfluency errors for the practiced native and practiced non-native 1091 sequences during training (outside the scanner) as a function of training block (1-8) for AWS only.

1092 The shaded area represents the standard error of the mean.

1093 Figure 3: Behavioral performance measures during test (inside the scanner) for the practiced 1094 native, practiced non-native, and novel non-native sequences as a function of as a function of test 1095 block (1-8) and group (ANS vs. AWS). (A) Mean percentage of errors of each sequence type; (B) 1096 Mean durations of the properly executed utterances of each sequence type; (C) Mean reaction 1097 times of the properly executed utterances of each sequence type. The shaded area represents the 1098 standard error of the mean.

1099 Figure 4: Mean percentage of disfluency errors (inside the scanner) for the practiced native, 1100 practiced non-native, and novel non-native sequences during test (inside the scanner) as a function 1101 of test block (1-8) for AWS only. The shaded area represents the standard error of the mean.

1102 Figure 5: Brain areas showing greater BOLD activation for novel non-native than practiced non1103 native trials. (A) Averaged across all 15 ANS and 16 AWS; (B) 15 ANS (left) and 16 AWS (right). 1104 Activation is displayed on a canonical inflated cortical surface. Colors indicate the relative 


\section{NEURAL CORRELATES OF SPEECH MOTOR SEQUENCE LEARNING}

1105 significance level at each voxel/vertex for the comparison of novel non-native and practiced non1106 native trials.

1107 Figure 6: Brain areas showing greater BOLD activation for practiced non-native than practiced 1108 native trials. (A) Averaged across all 15 ANS and 16 AWS; (B) 15 ANS (left) and 16 AWS (right).

1109 Activation is displayed on a canonical inflated cortical surface. The significant subcortical cluster

1110 is shown on a slice through the cerebellum at $\mathrm{z}=-54$ in the MNI template (bottom right); left and 1111 right hemispheres are indicated by $\mathrm{L}$ and $\mathrm{R}$, respectively. Colors indicate the relative significance 1112 level at each voxel/vertex for the comparison of novel non-native and practiced non-native trials.

1113 Figure 7: Significant correlation between participant-normalized difference in mean error rates 1114 between the novel non-native and practiced non-native words and the mean activation in left aINS $1115[r=.381, p=.034]$ (left panel) and right aINS $[r=.404, p=.024]$ (right panel).

1116 Figure 8: Significant correlation between mean disfluency rates during the test phase (inside the

1117 scanner) and mean activation in left GPe $[r=-0.609, p-F D R=.047]$ (left panel) and left caudate $1118[r=-.591, p-F D R=.047]$ (right panel). 
NEURAL CORRELATES OF SPEECH MOTOR SEQUENCE LEARNING

\section{Table captions}

1121 Table 1. International phonetic alphabet (IPA) transcription and orthography for experimental

1122 stimuli used to elicit the native (left) and non-native (right) target onset and coda clusters 1123 (underlined).

1124 Table 2. List of the predefined cortical and subcortical regions-of-interest (ROIs) used in the 1125 current analyses.

1126 Table 3. Summary of the ANOVA results for the behavioral analyses. Shown are the $F$ value, the 1127 degrees of freedom, $p$ value, and partial-eta-squared value for each effect.

1128 Table 4. Mean error rates in the test blocks (inside the scanner) by error subtype and sequence 1129 type.

1130 Table 5. Summary of significant cortical and subcortical activation peaks for the novel non-native

1131 - practiced non-native contrast. From left to right, the columns show the anatomical region 1132 name(s), number of peaks, MNI stereotactic coordinates, cluster size, TFCE value, and pFw value. 1133 Cluster regions are listed in descending order of number of significant vertices.

1134 Table 6. Summary of significant cortical and subcortical activation peaks for the practiced non1135 native - practiced native syllable contrast. From left to right, the columns show the anatomical 1136 region name(s), number of peaks, MNI stereotactic coordinates, cluster size, TFCE value, and $p_{F W E}$ 1137 value. Cluster regions are listed in descending order of number of significant vertices. 
ANS

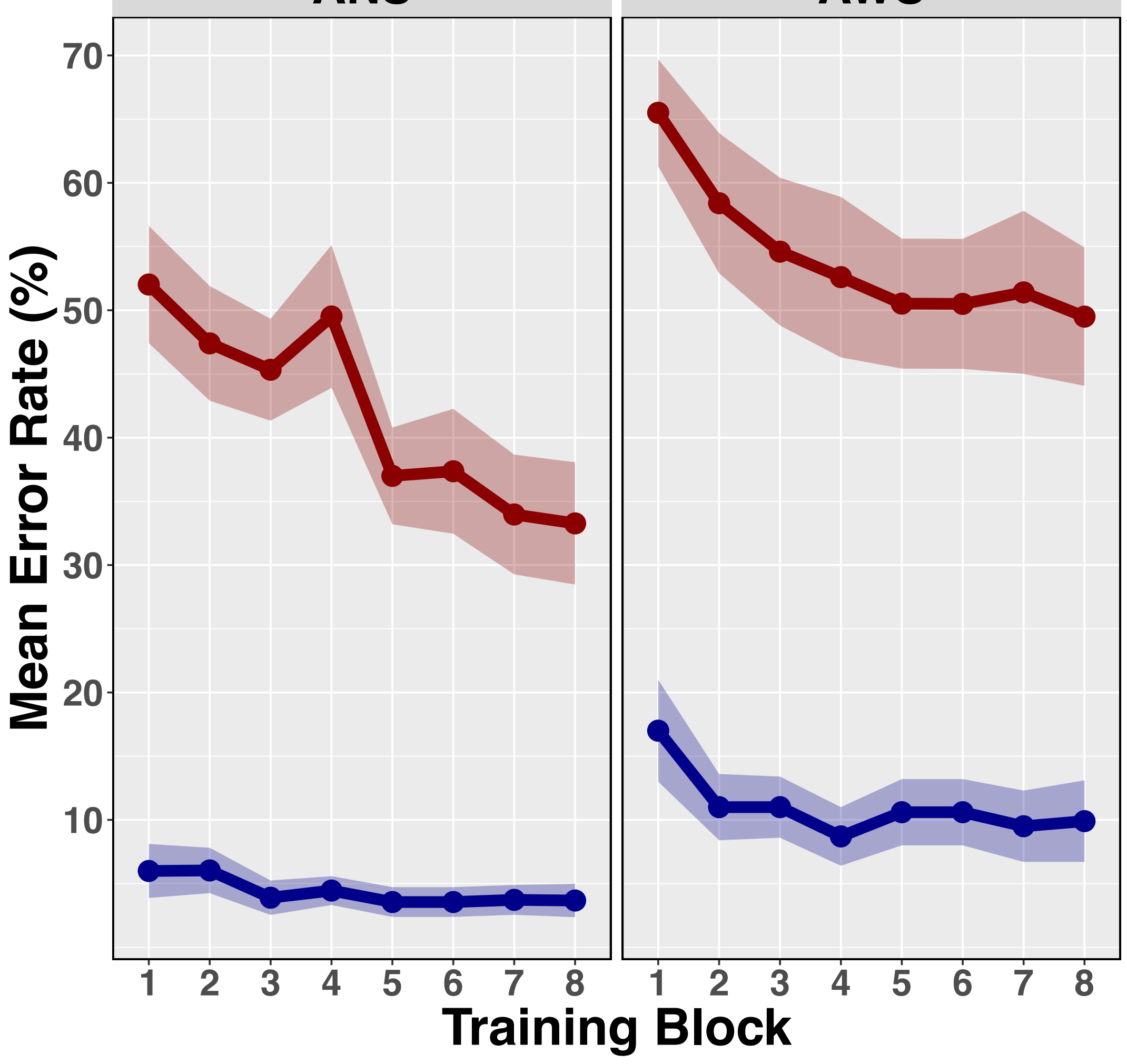

Sequence Type

- Practiced Native

- Practiced Nonnative 
ANS

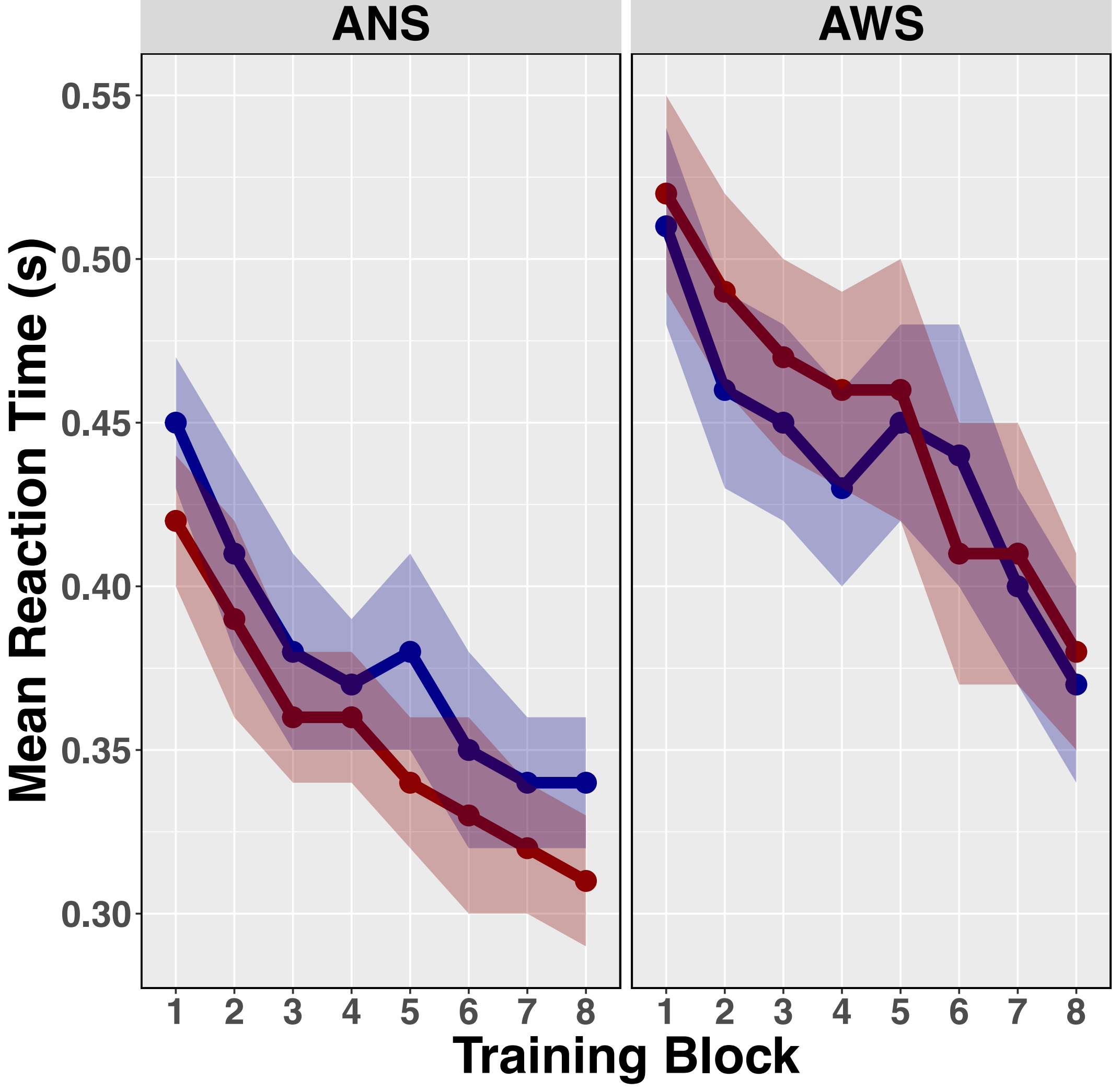

Sequence Type

- Practiced Native

Practiced Nonnative 


\section{ANS}

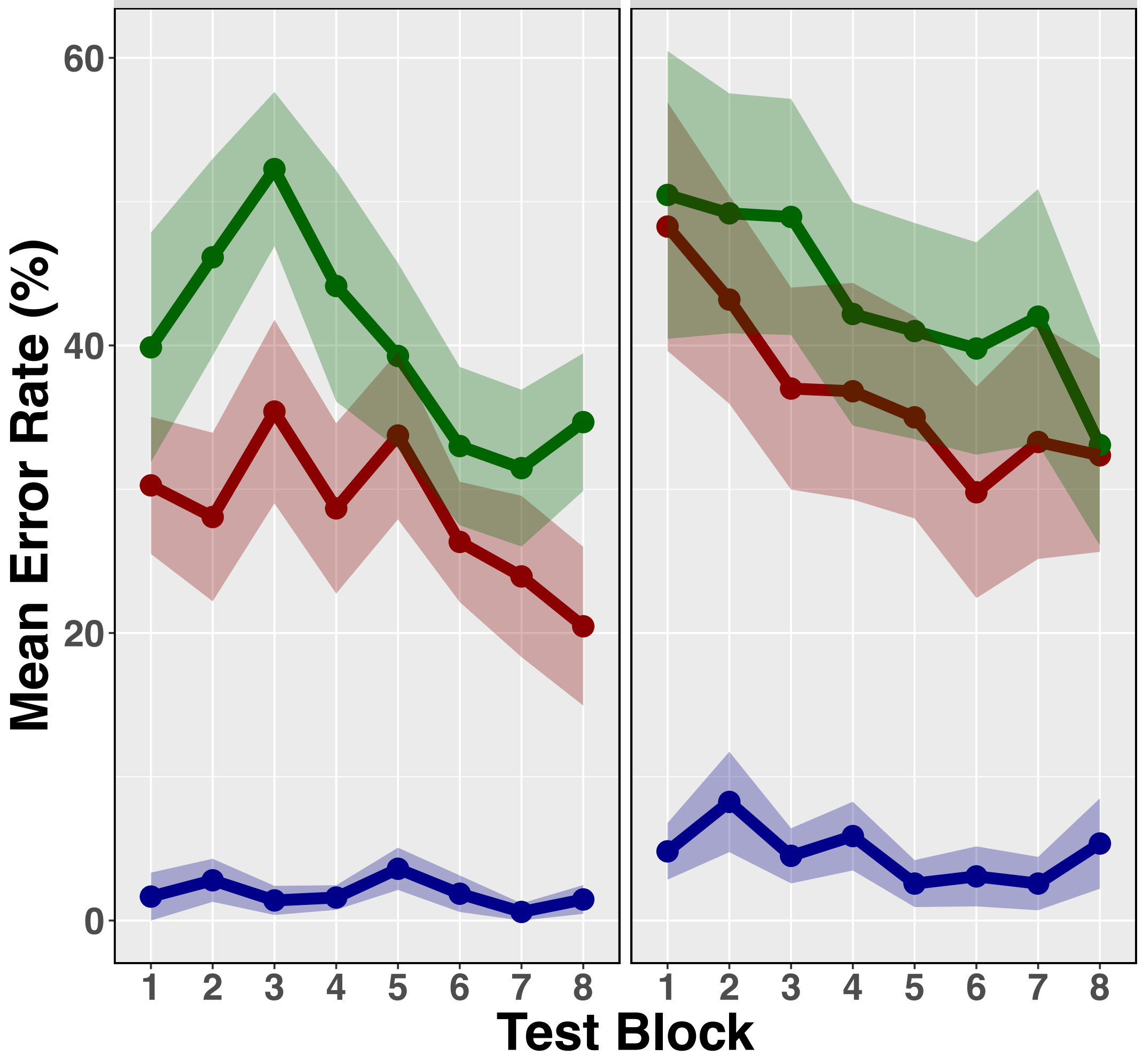

Sequence Type

- Practiced Native Practiced Nonnative Novel Nonnative 
ANS

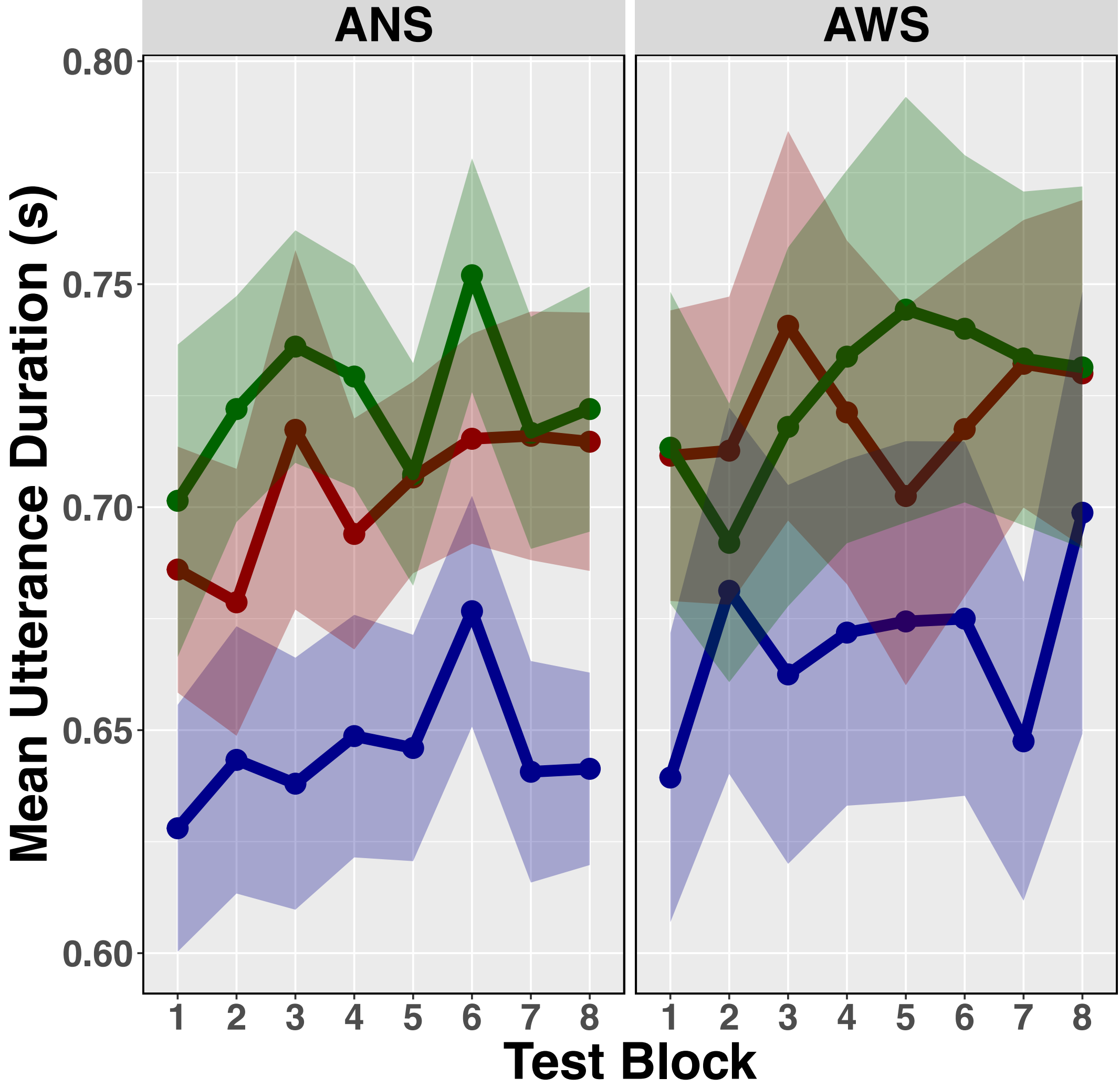

Sequence Type

- Practiced Native

Practiced Nonnative Novel Nonnative 


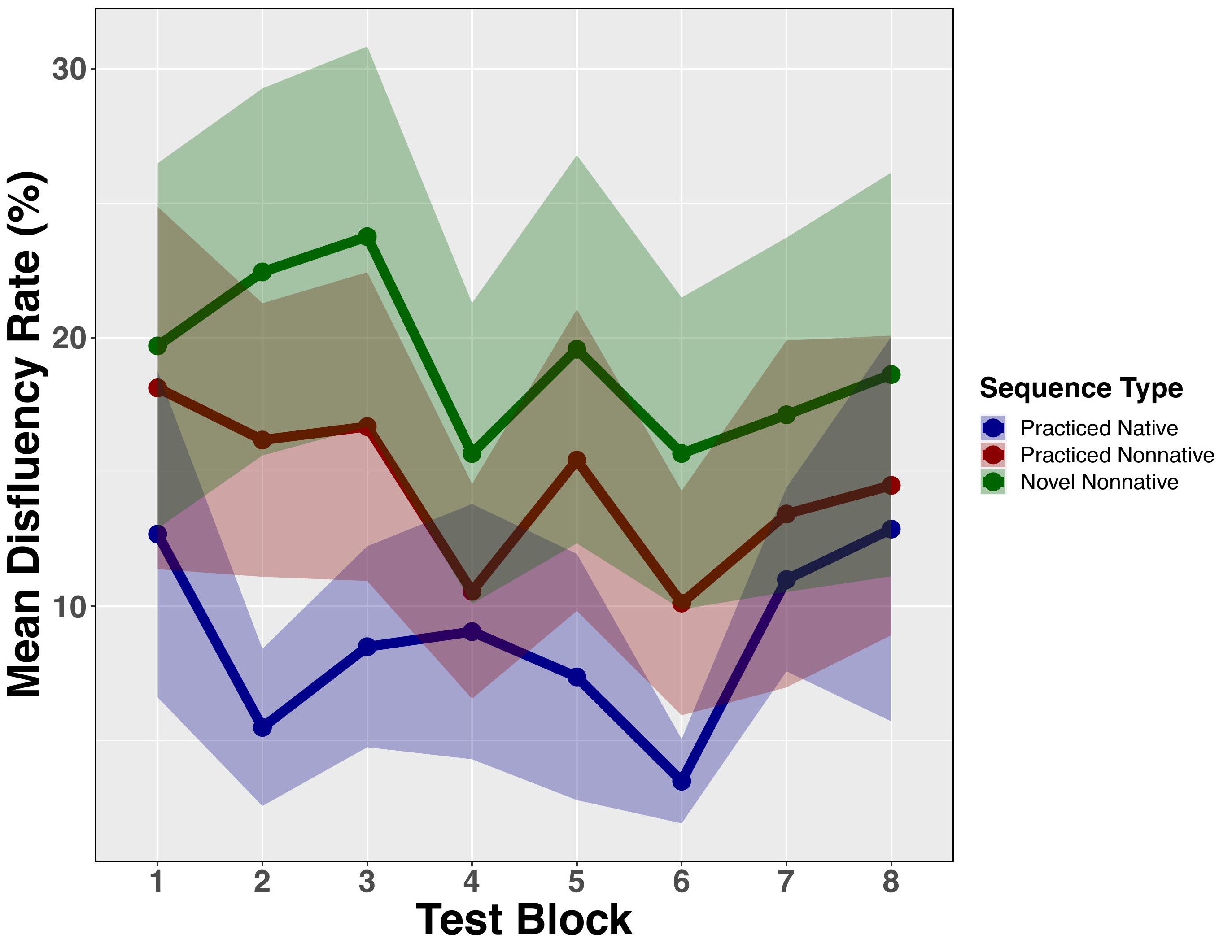



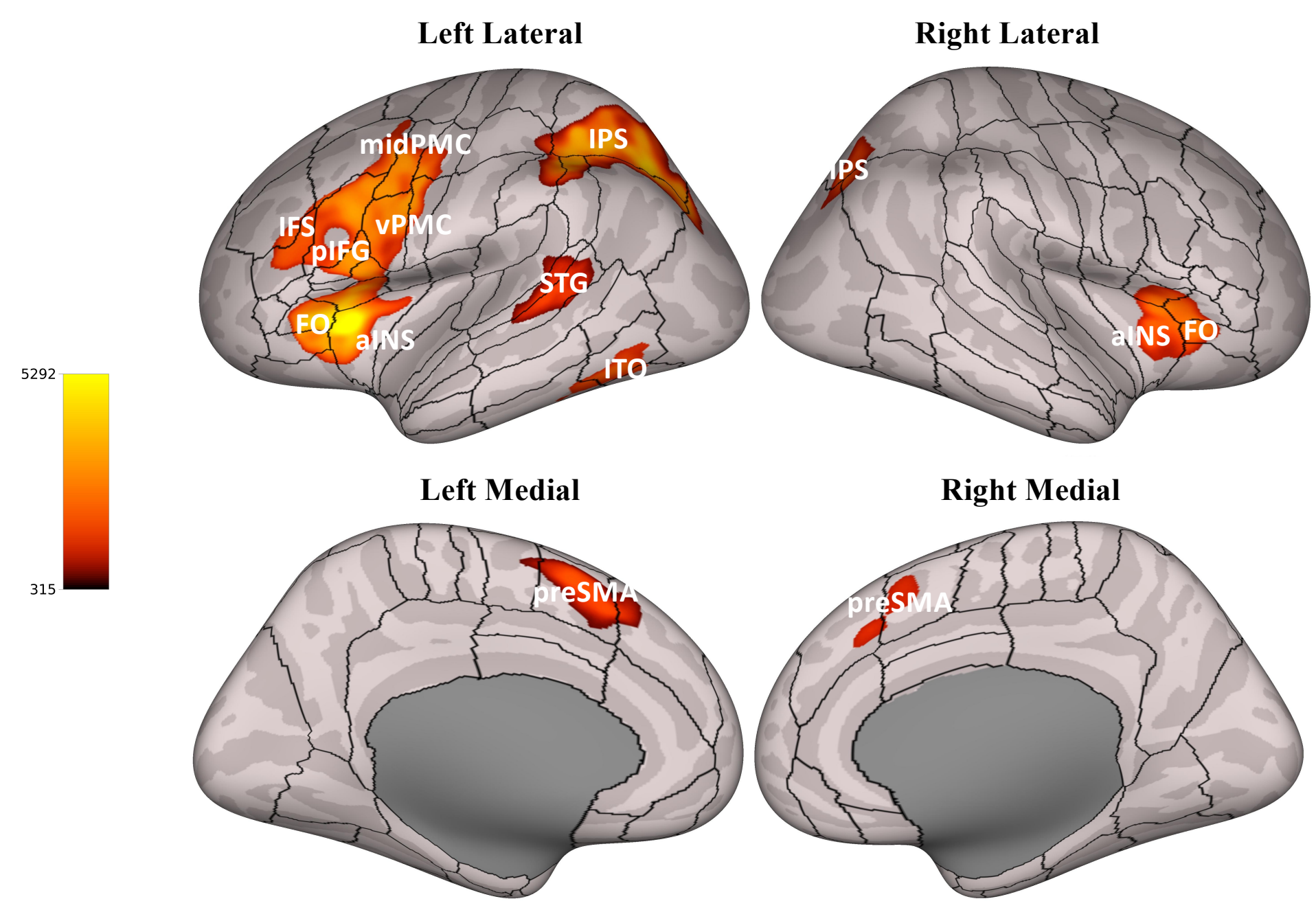
ANS
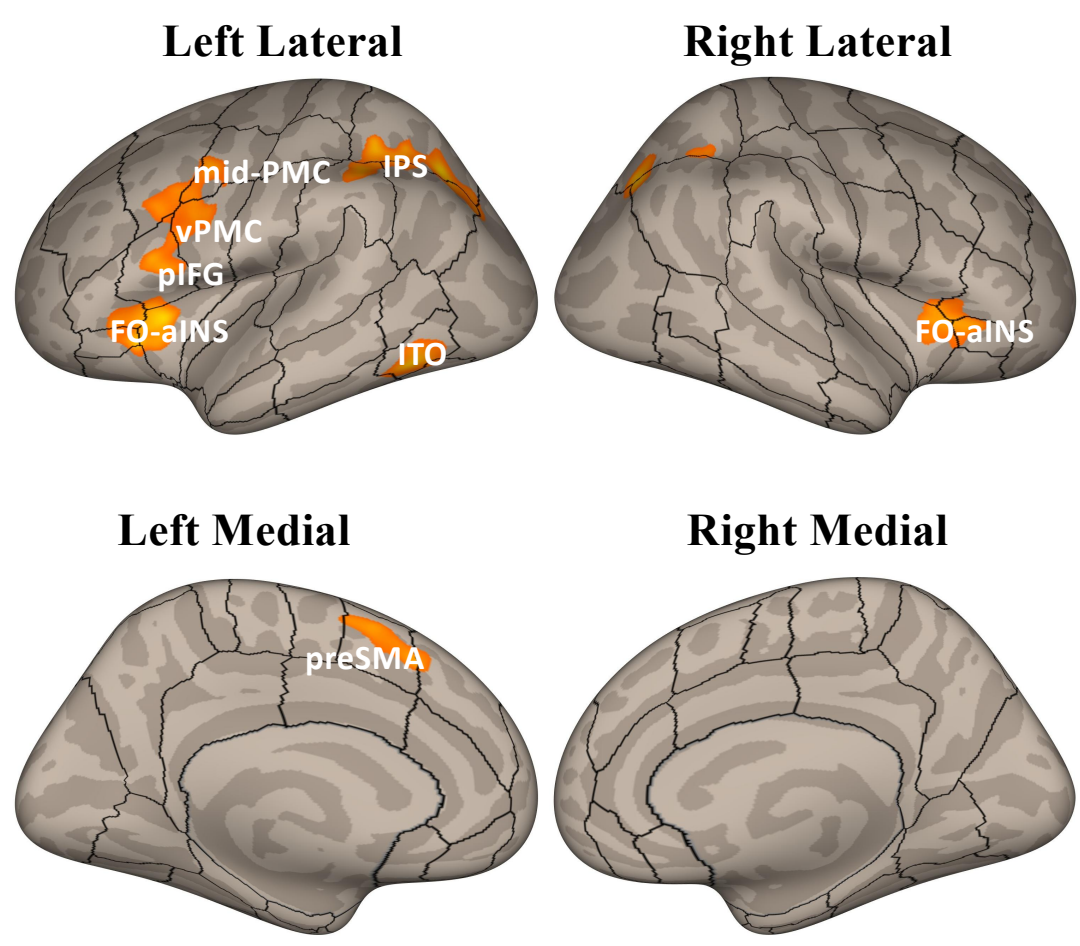

Right Medial

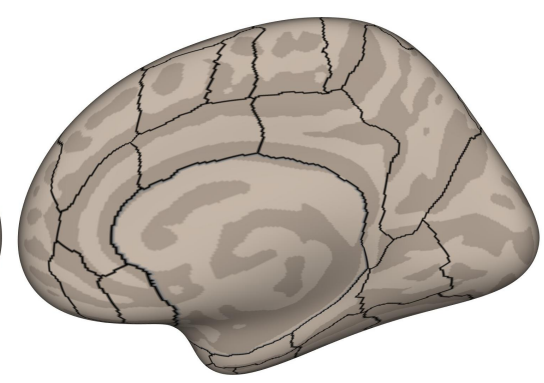

AWS
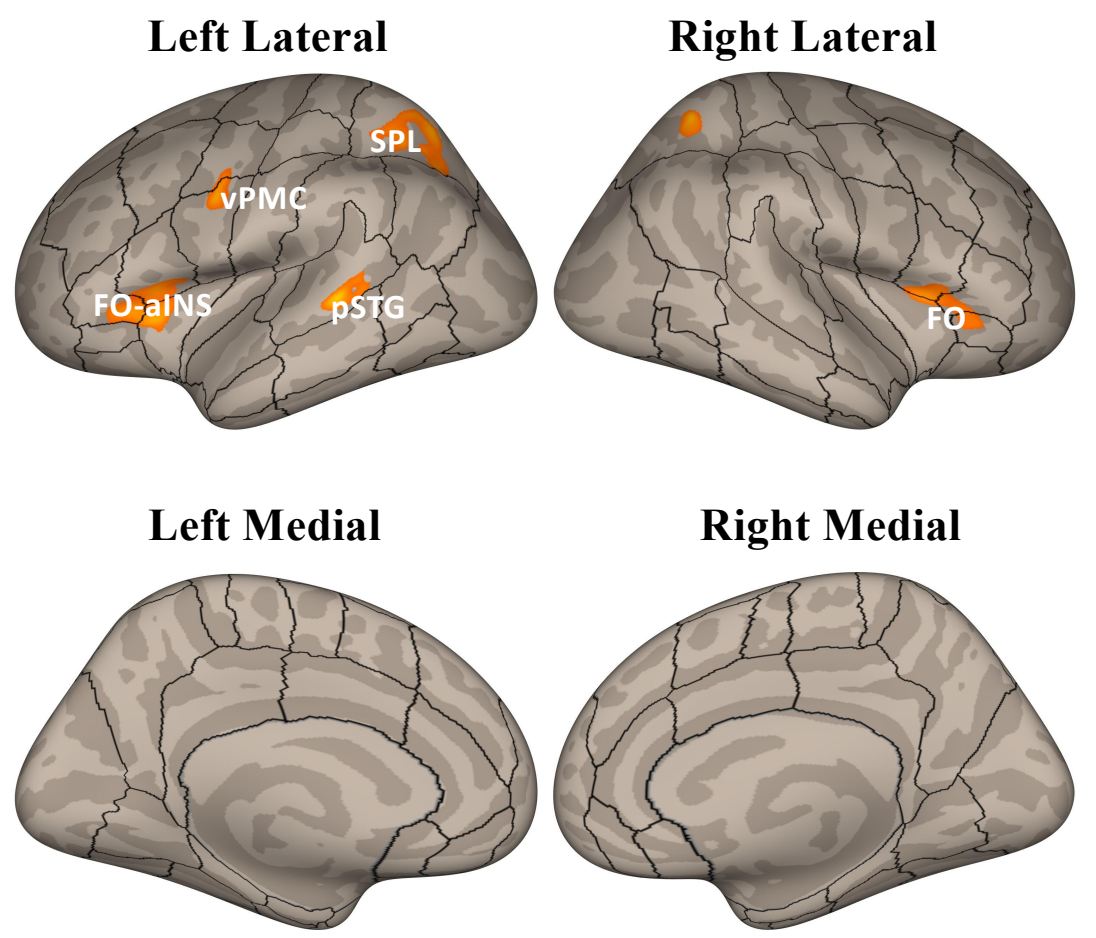

Right Medial

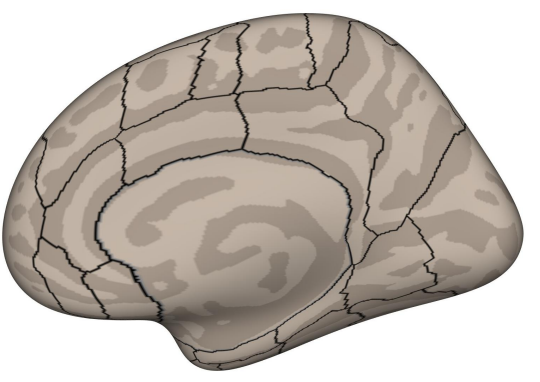



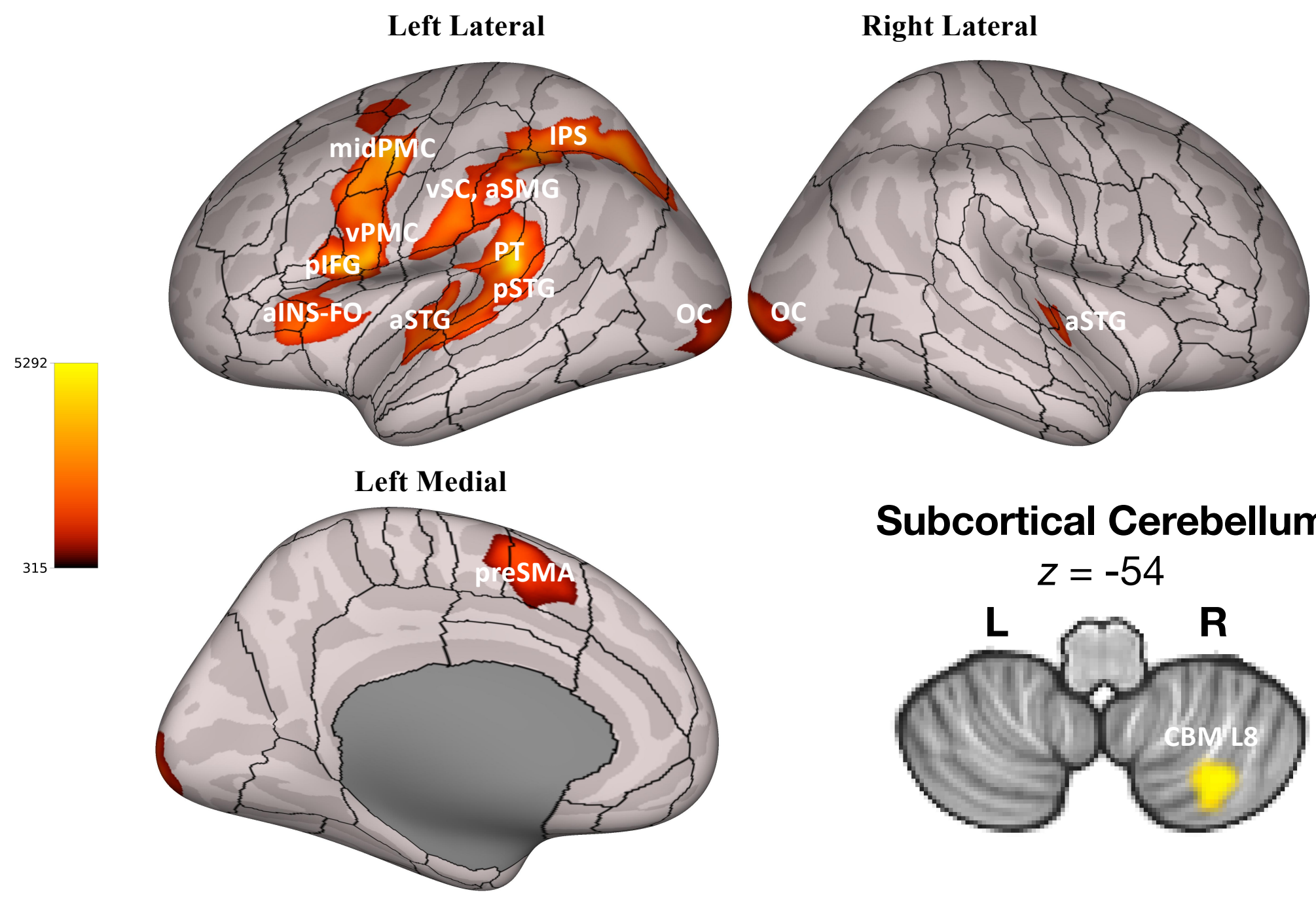

\section{Subcortical Cerebellum}

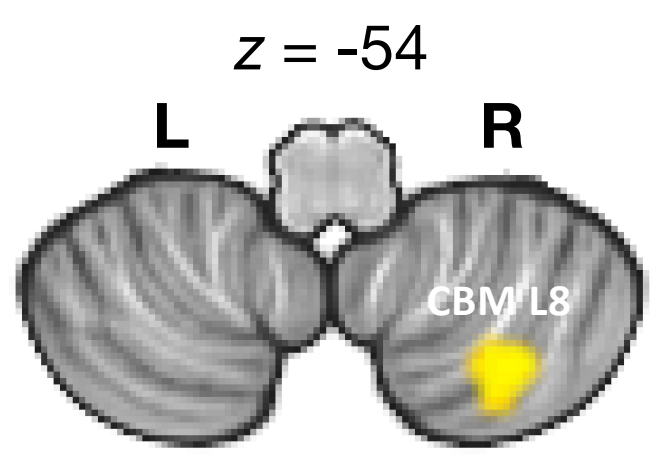


ANS
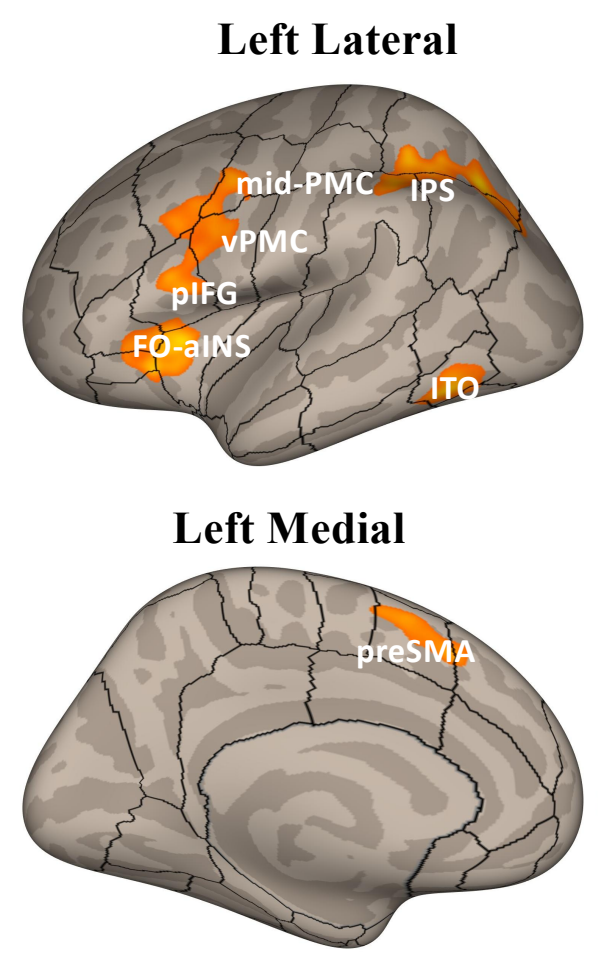

Right Lateral

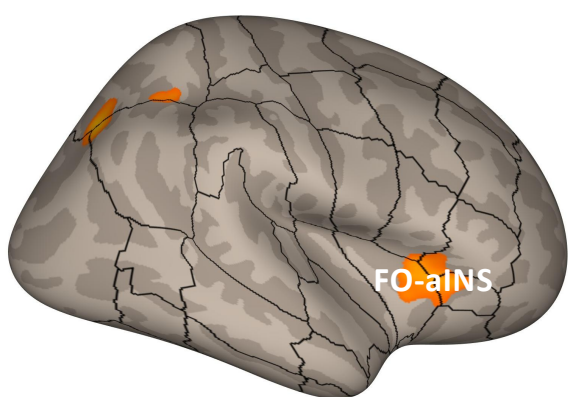

Right Medial

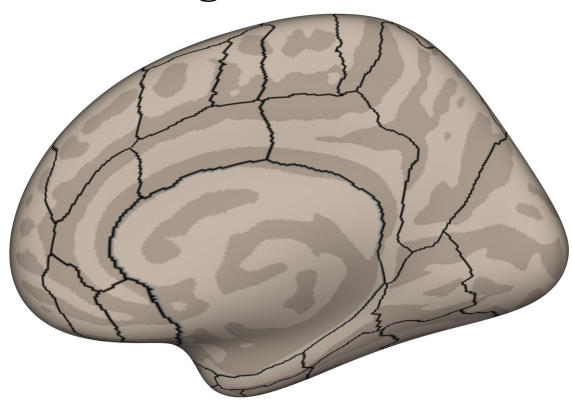

AWS
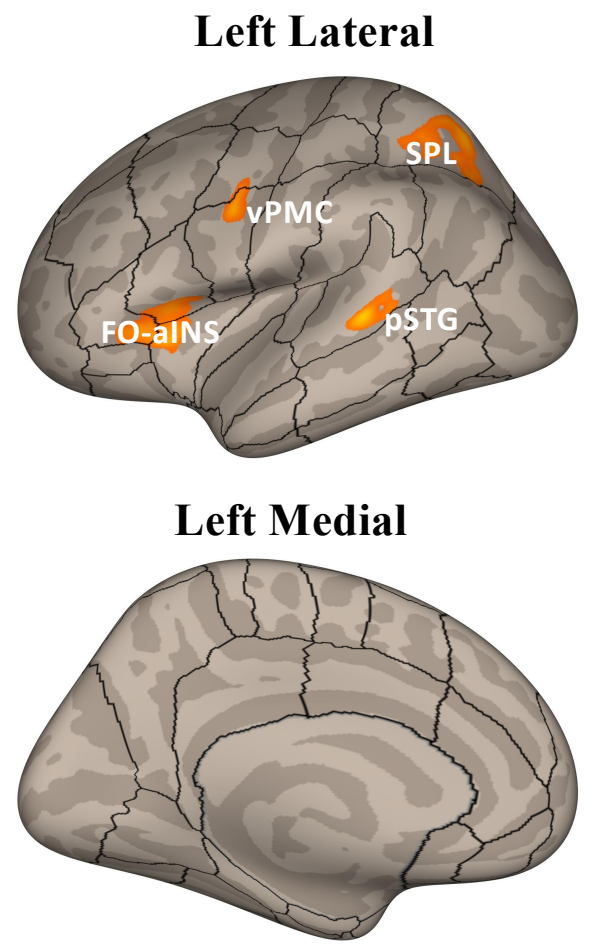

Right Lateral

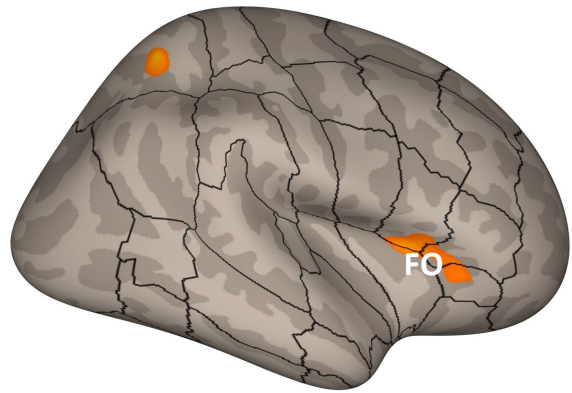

Right Medial

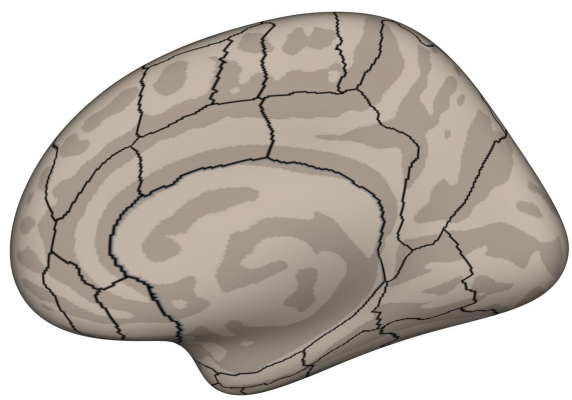



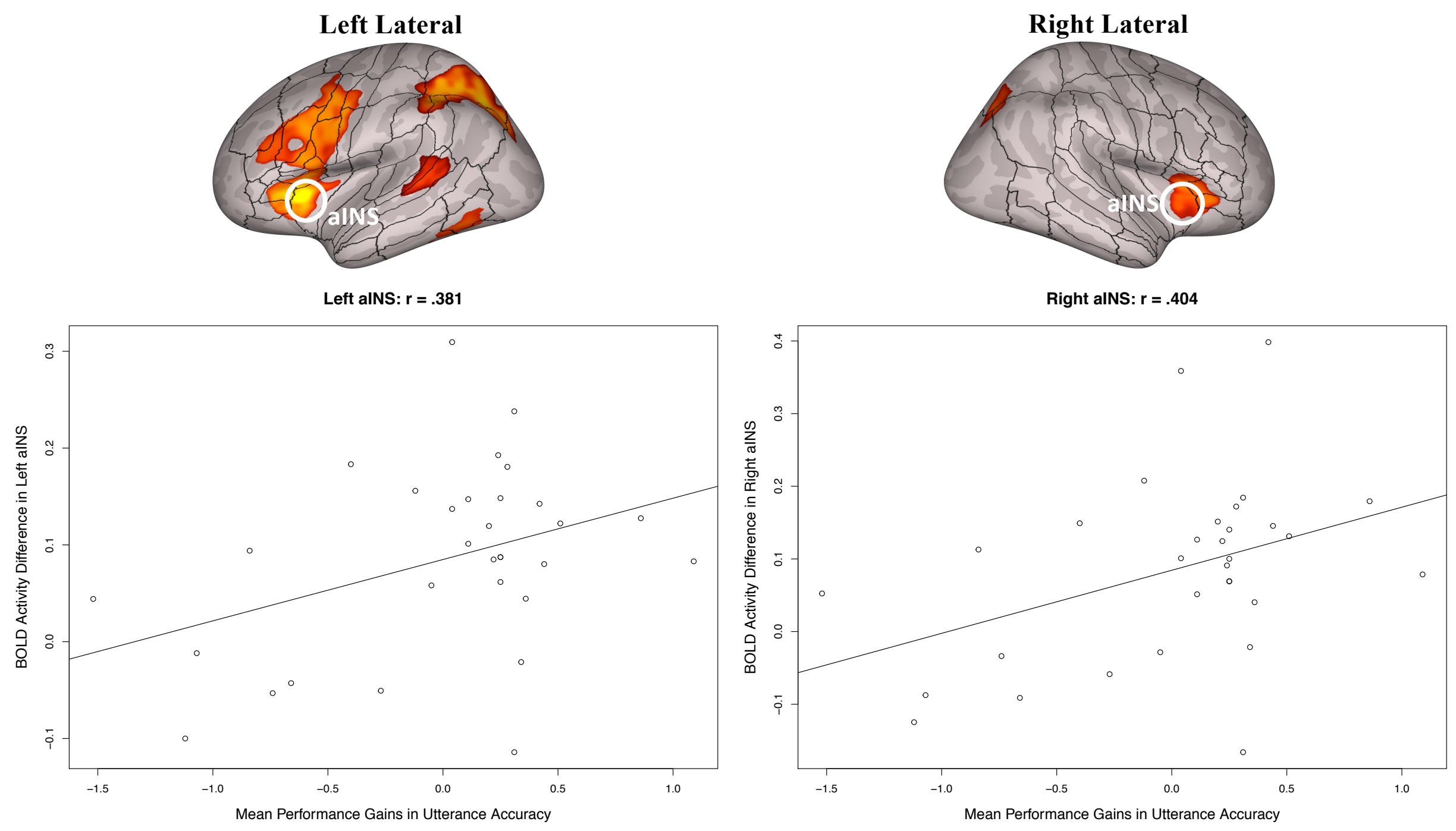

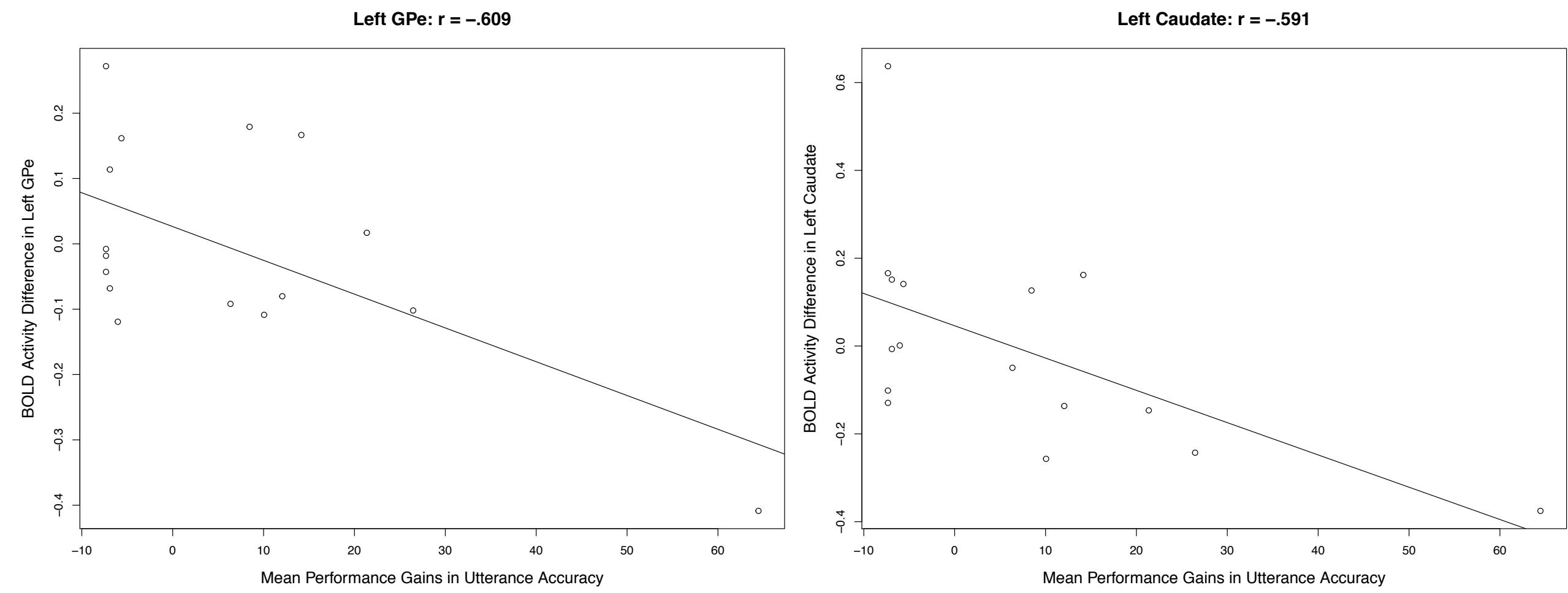


\title{
Supplementary Material for:
}

\section{Neural correlates of speech motor sequence learning in stuttering vs. nonstuttering adults: an fMRI investigation}

\author{
Matthew Masapollo ${ }^{1}$, Jennifer A. Segawa ${ }^{1,2}$, Deryk S. Beal ${ }^{1,3}$, Matthias Heyne ${ }^{1}$, Jason A. \\ Tourville $^{1}$, Alfonso Nieto-Castañón ${ }^{1}$, Saul Frankford ${ }^{1}$, \& Frank H. Guenther ${ }^{1,4,5}$ \\ ${ }^{1}$ Department of Speech, Language and Hearing Sciences, Boston University, Boston, MA 02215 \\ ${ }^{2}$ Departments of Neuroscience and Biology, Stonehill College, Easton, MA 02357 \\ ${ }^{3}$ Department of Speech-Language Pathology, University of Toronto, Toronto, ON M5G 1V7 \\ ${ }^{4}$ Department of Biomedical Engineering, Boston University, Boston, MA 02215 \\ ${ }^{5}$ Department of Radiology, Massachusetts General Hospital, Charlestown, MA 02129
}

\section{S1. Given the differences in reaction time (RT) scores between the ANS and AWS groups, is the timing assumption of the sparse sampling protocol valid?}

As shown in Figure 3C, the RT scores were approximately 200 ms longer in AWS than in ANS. These delayed responses were unlikely to have an effect on the obtained BOLD signal in the test phase given i.) the width of the delay of the hemodynamic response, and ii) that task performance is well under one second (as shown in Figure 3B). Nevertheless, in order to address the possibility that some trials containing "silent" stuttering blocks were included in the neuroimaging analyses, we here provide supplementary data showing the distribution of RT scores for the AWS group (Figure S2) as a whole and for each individual stuttering speaker (Figure S3) in the test phase. Critically, these plots did not reveal a bimodal distribution with one peak having frequency values clustered to the right. This finding suggests that any potential stuttering blocks or pauses were likely categorized as silent errors (sequencing subtype 4 as described; see main text for explanation) and were excluded from the imaging analyses, in which case, the BOLD responses would not have been contaminated by potential delays in RT. 
S2. Figures for Supplementary Material

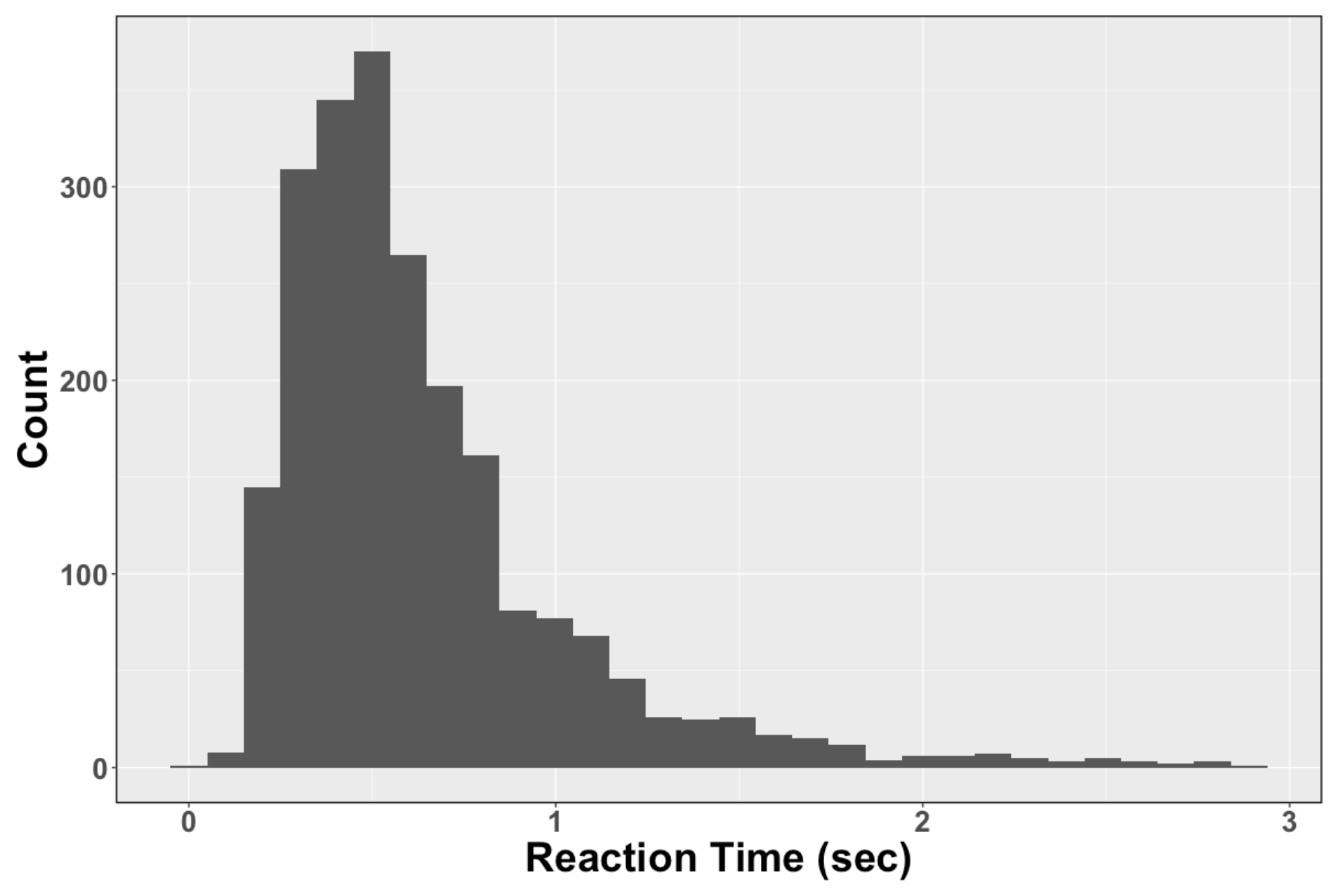

Figure S1: Histogram plots of reaction time (RT) measures (30 ms bins) for the properly sequenced productions (pooled across experimental conditions and AWS participants) during the test phase (inside the scanner). Note that the audio recording window was three seconds in duration (starting from the offset of the "go" signal). 


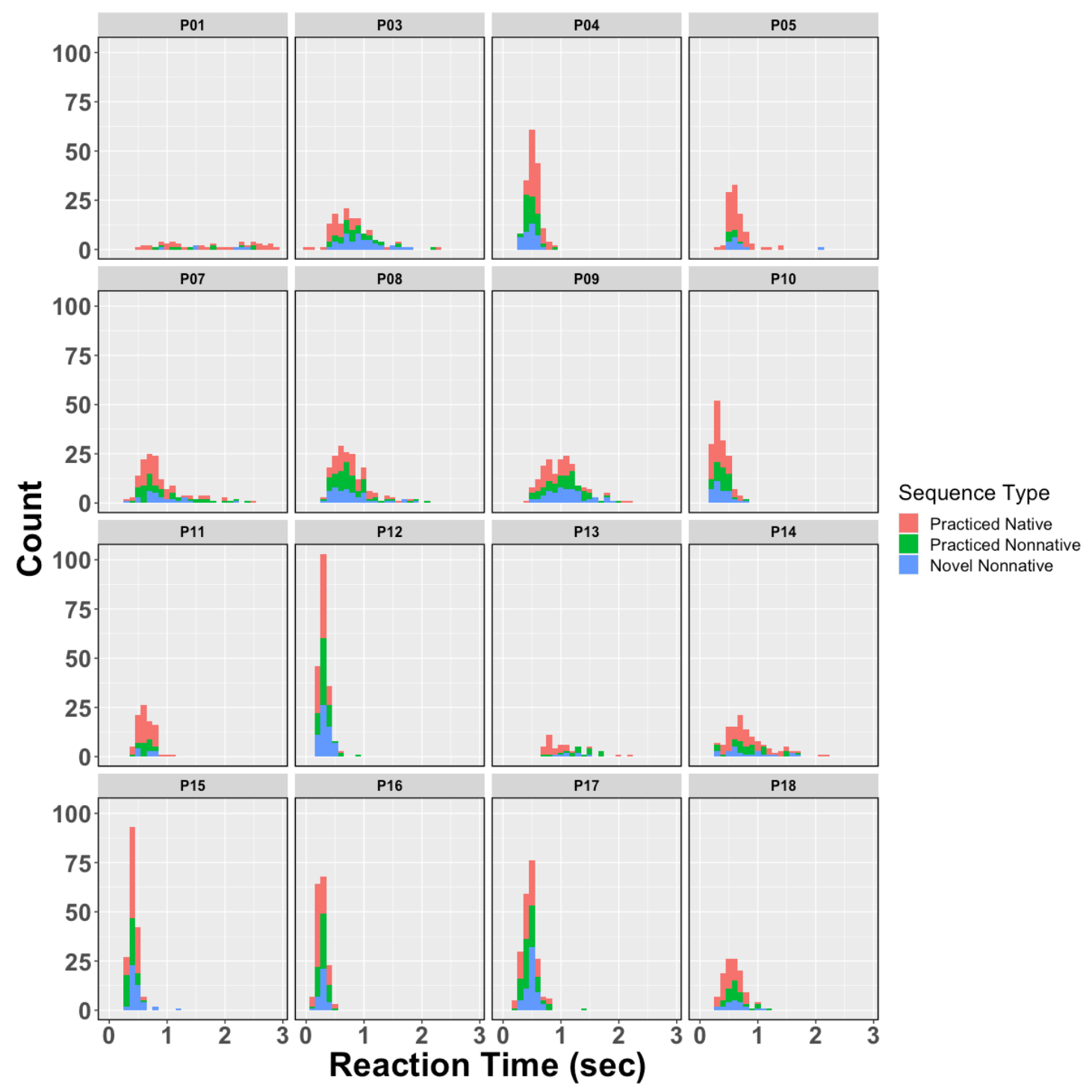

Figure S2: Histograms plots of reaction time (RT) measures (30 ms bins) for the properly sequenced productions during the test phase (inside the scanner) for each participant in the AWS group. The counts are plotted as a function of experimental condition (practiced native vs. practiced nonnative vs. novel nonnative) and participant $(\mathrm{n}=16) . \mathrm{P}=$ patient. 


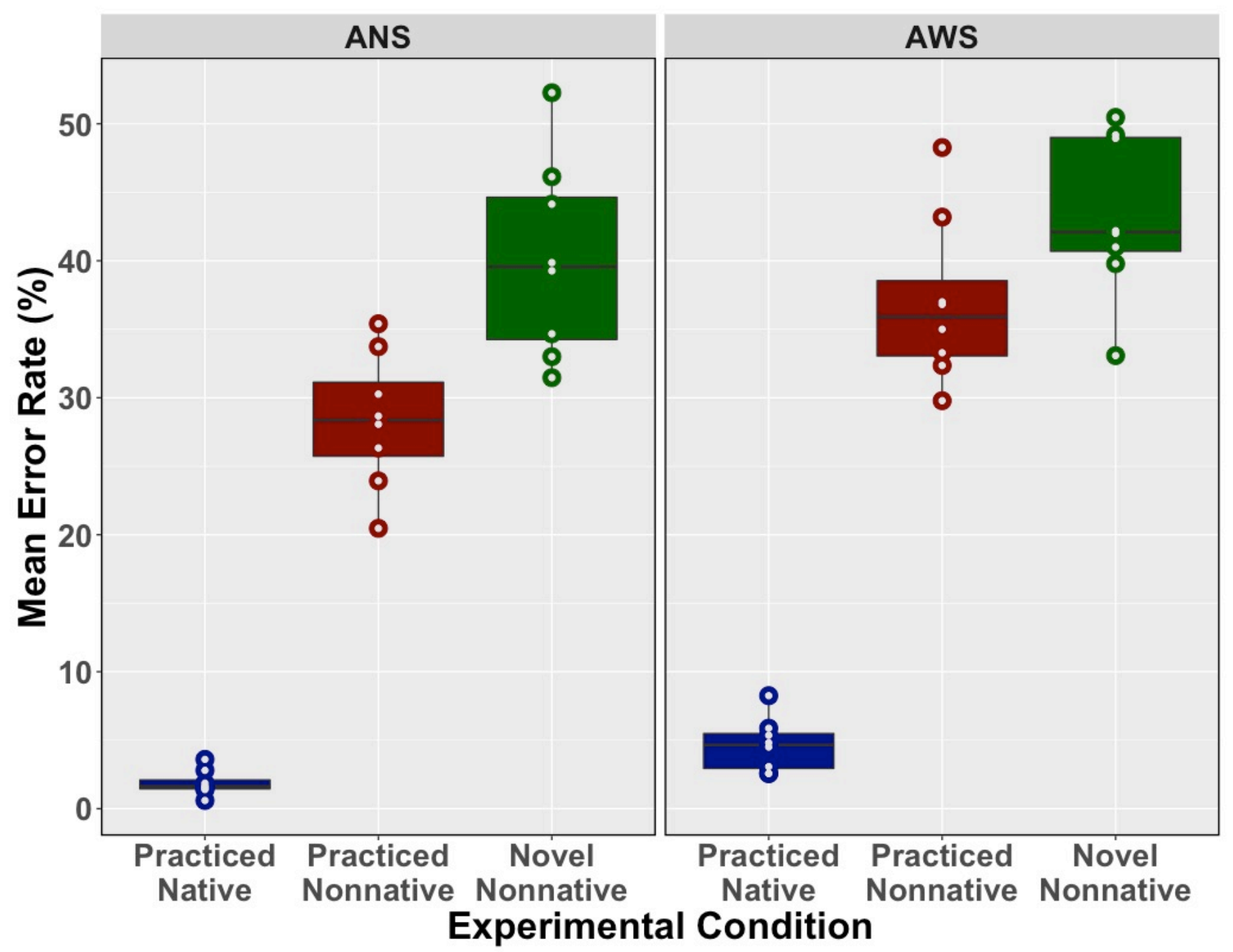

Figure S3: Boxplots of mean percentage of errors during the test phase (inside the scanner) as a function of experimental group (ANS vs. AWS) and sequence type (practiced native vs. practiced non-native vs. novel non-native) 


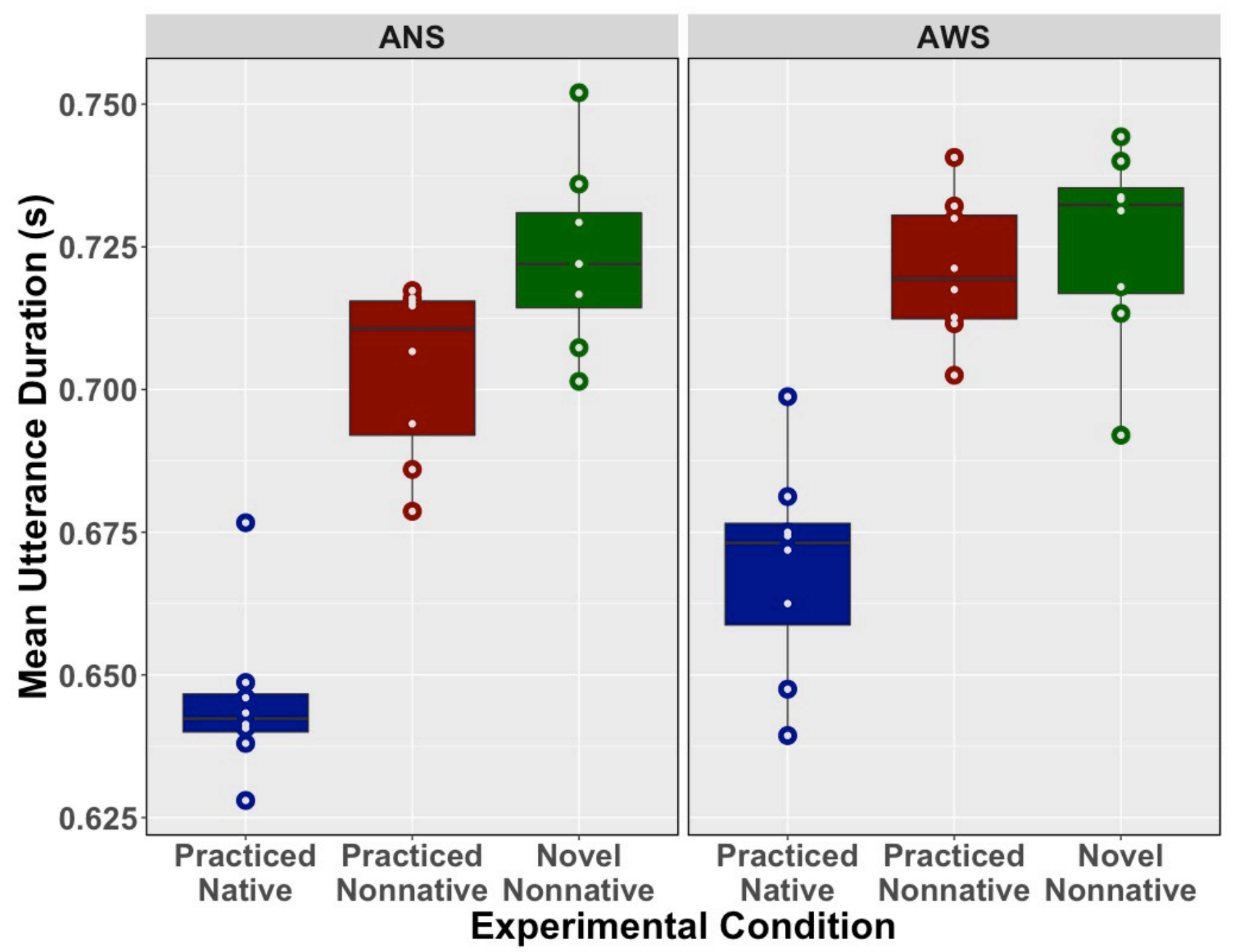

Figure S4: Boxplots of mean utterance durations (for properly sequenced productions only) during the test phase (inside the scanner) as a function of experimental group (ANS vs. AWS) and sequence type (practiced native vs. practiced non-native vs. novel non-native). 


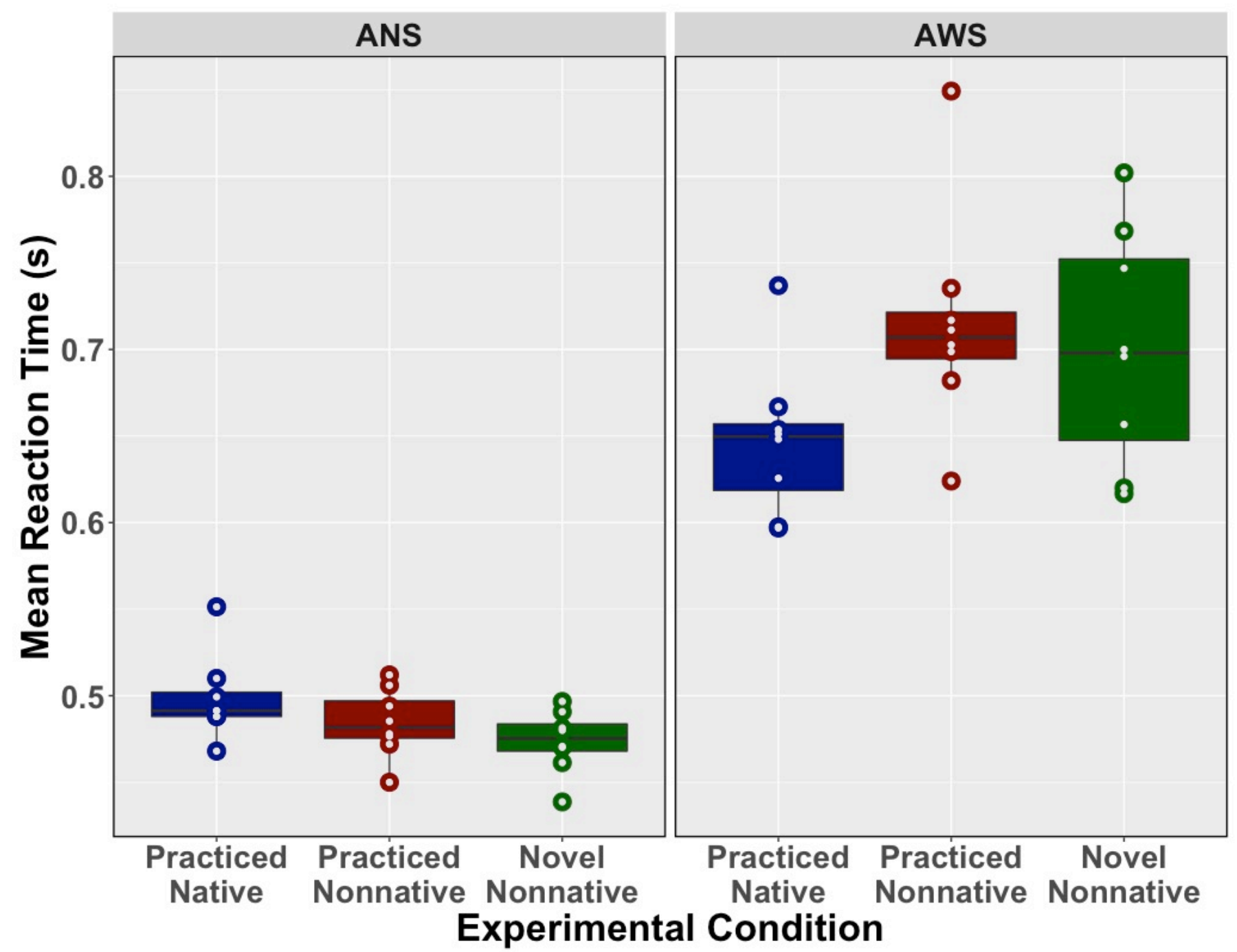

Figure S5: Boxplots of mean reaction times (for properly sequenced productions only) during the test phase (inside the scanner) as a function of experimental group (ANS vs. AWS) and sequence type (practiced native vs. practiced non-native vs. novel non-native). 


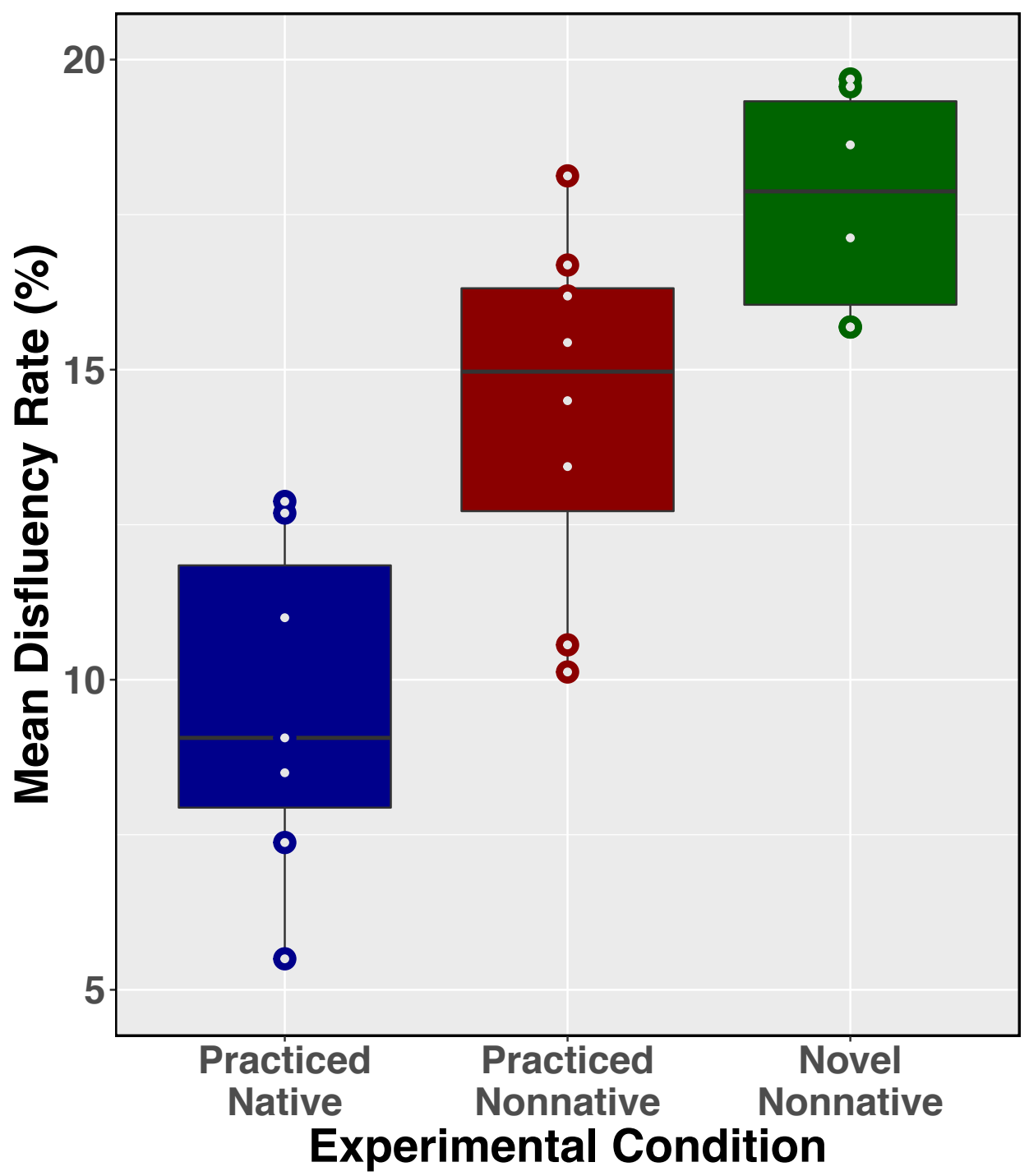

Figure S6: Boxplots of mean percentage of disfluencies during the test phase (inside the scanner) as a function of sequence type (practiced native vs. practiced non-native vs. novel non-native) for the AWS group only. 